\title{
1 Post-transcriptionally regulated genes are essential for pluripotent stem cell
}

\section{2 survival}

Mio Iwasaki ${ }^{1,7 *}$, Yuka Kawahara ${ }^{1}$, Tsuyoshi Tabata ${ }^{1}$, Yohei Nishi, ${ }^{1,2}$, Megumi Narita1, Akira Ohta1, Hirohide Saito ${ }^{1}$, Takuya Yamamoto ${ }^{1,3,4}$, Masato Nakagawa ${ }^{1}$, Shinya

1Department of Life Science Frontiers, Center for iPS Cell Research and Application,

9 Kyoto University, Kyoto 606-8507, Japan

$10 \quad{ }_{2}^{2}$ PSC-Based Drug Discovery and Development Team, RIKEN Bio Resource 11 Research Center (BRC), Kyoto, Japan.

12 3Institute for the Advanced Study of Human Biology (WPI-ASHBi), Kyoto

13 University, Kyoto 606-8501, Japan

$14{ }^{4}$ Medical-risk Avoidance based on iPS Cells Team, RIKEN Center for

15 Advanced Intelligence Project (AIP), Kyoto 606-8507, Japan

16 5Gladstone Institute of Cardiovascular Disease, San Francisco, CA 94158, USA

$17{ }^{6}$ Department of Anatomy, University of California, San Francisco, San Francisco, CA

18 94143, USA

19 'Lead Contact

$20{ }^{*}$ Correspondence: omio@cira.kyoto-u.ac.jp (M.I.), kazu@cira.kyoto-u.ac.jp (K.T.)

\section{Keywords}

23 Pluripotent stem cells, post-transcriptional regulation, proteomics, transcriptomics

\section{Summary}

26 The effects of transcription factors on the maintenance and differentiation of

27 pluripotent stem cells (PSCs) have been well studied. However, the importance of post-transcriptional regulatory mechanisms, which cause the quantitative dissociation of mRNA and protein expression, has not been explored in detail. Here, 
1 by combining transcriptome and proteome profiling, we identified 228 post-

2 transcriptionally regulated genes with strict upregulation of the protein level in PSCs.

3 Among them, we found that 84 genes were vital for the survival of PSCs and HDFs,

4 including 20 genes that were specifically necessary for the survival of PSCs. These

520 proteins were upregulated only in PSCs and not in differentiated cells derived

6 from the three germ layers. Subcellular fractionation of the mRNA showed that the

7 expression of most of the 20 proteins was regulated at the mRNA localization stage

8 from the nucleus to the cytoplasm, but their translation efficiency was constant.

9 Together, these results revealed that post-transcriptionally regulated genes have a

10 crucial role in PSC survival.

\section{INTRODUCTION}

13 The processing (Bentley, 2014), export (Hentze et al., 2018), turnover (Dendooven

14 et al., 2020), and accurate decoding of mRNA (Teixeira and Lehmann, 2019),

15 ribosome biogenesis (Pelletier et al., 2018), and protein degradation (Pohl and Dikic,

16 2019) are critical steps in the post-transcriptional regulation of gene expressions.

17 Among the several hundred human cell types, pluripotent stem cells (PSCs) have

18 unique post-transcriptional mechanisms (Chen and $\mathrm{Hu}, 2017$ ), such as specific

19 mRNA processing events, that establish and maintain the pluripotent state (Gabut et

20 al., 2011; Han et al., 2013; Ohta et al., 2013; Salomonis et al., 2010). Interestingly,

21 for many genes, differences in protein levels in PSCs are not accompanied by

22 differences in the corresponding mRNA levels (Lu et al., 2009), suggesting post-

23 transcriptionally regulated genes exist in strict regulation at the protein level. One

24 example is the CLOCK gene, in which the amount of mRNA is constant before and

25 after differentiation, whereas the protein expression depends on the cell type and is

26 absent in PSCs (Umemura et al., 2017). Considering that protein levels are more

27 conserved than mRNA levels in primates (Khan et al., 2013), the strict regulation at

28 the protein level with constant mRNA quantities might have important effects on

29 pluripotency and differentiation during development. 
1 To systematically identify genes differently regulated at the mRNA and protein level,

2 transcriptome analysis by RNA sequencing or microarray and proteome analysis by mass spectrometry (MS) have been performed on various cell types (Buccitelli and Selbach, 2020; Matsumoto et al., 2017; Roumeliotis et al., 2017; Wang et al., 2019).

5 Typically, a high correlation in the levels is shown for core metabolic pathway-related genes, but a low correlation is observed for ribosomal and spliceosome genes. However, whether post-transcriptionally regulated genes with tightly controlled protein levels are essential for the maintenance of the cell remains an open question.

9 Here, we compared global mRNA and protein levels between PSCs and

10 differentiated cells to identify essential post-transcriptionally regulated genes. We

11 found 228 post-transcriptionally regulated genes exclusively in PSCs that showed

12 specific biological functions for RNA and nucleic acid binding. siRNA screening

13 revealed that $54 \%$ (84 genes) were necessary for cell survival and 20 of them were

14 identified as PSC-specific. Finally, we showed that these 20 genes are mainly 15 regulated at the mRNA localization stage rather than the translation stage, 16 suggesting an importance of cell type-specific post-transcriptional regulation in 17 PSCs.

\section{RESULTS}

\section{8 upregulated proteins in PSCs showed constant mRNA expression}

21 First, to identify upregulated genes and post-transcriptionally regulated genes, we 22 analyzed the mRNA and protein levels by microarray and MS, respectively (Figure 23 1A). We used two human dermal fibroblast (HDF) lines (HDF-1, HDF1388; and HDF24 2, Tig120), two human induced PSC (iPSC) lines (iPSC-1, 201B7; and iPSC-2, 25 1418E1), and one human embryonic stem cell line (ESC, H9). We compared the 26 ratio of the mRNA and protein levels for 6404 genes for all combinations of PSCs 27 (iPSC-1, -2, ESC) and HDFs (HDF-1, -2) (Figure S1). In the six pairs of different cell types, commonly upregulated genes were defined as having at least a 1.9-fold change $\left(\log _{2}(0.9)\right)$. We found that 1275 genes had upregulated mRNA levels in all 
1 PSCs, and 1468 genes had upregulated protein levels (Figure 1B). Among these

2 genes, 509 showed upregulated levels for both mRNA and protein, including PSC-

3 specific genes, such as LIN28A, OCT3/4, and SOX2 (Figure 1B, C). Regarding

4 HDFs, the numbers were 1519 and 461, respectively, and the number with common

5 upregulation was 387, including HDF-specific genes, such as FN1 and VIM (Figure

$61 \mathrm{~B}, \mathrm{C})$. Overall, each cell-specific gene showed a highly correlated mRNA to protein

7 ratio (Figure $1 \mathrm{C}$ and Table S1).

8 Next, we examined genes that were post-transcriptionally regulated by selecting

9 those that were upregulated only at the mRNA or protein level by $\log _{2}(0.9)$ (Figure

10 1C; see Trans-omics data analysis in Methods). From this analysis, we found 10 and

1156 genes in PSCs and HDFs, respectively, upregulated only at the mRNA level, and

12228 and 8 genes upregulated only at the protein level. To investigate the function of

13 these four gene sets, we conducted a Gene Ontology (GO) analysis (Fig. 1D).

14 Especially among the 228 genes in PSCs that showed only upregulated protein

15 levels, we found specific molecular functions, such as RNA binding and nucleic acid

16 binding. Although the 8 genes for HDFs showed no enrichment in molecular

17 functions, we named these 228 and 8 genes "upregulated protein levels independent

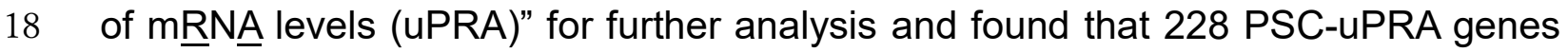

19 have specific nucleic acid binding functions.

2120 uPRA genes are essential for PSC cell survival

22 To investigate which uPRA genes are significant for cell maintenance, we performed

23 siRNA knockdown screening in iPSC-1, -2, and HDF-2 (Figure 2A, 2B, Table S2, S3).

24 We picked siRNAs to target 156 (Table S2) of the 228 PSC-uPRA and 8 HDF-uPRA

25 genes. The siRNA knockdown of OCT3/4 and LaminB2 was respectively used as a

26 pluripotency marker for PSCs and a universal control for both cell types (Figure 2B).

27 The knockdown of none of the 156 uPRA genes induced differentiation (Table S3).

28 On the other hand, the knockdown of 84 of the 156 uPRA genes, such as SRRT and

29 RSL1D1, significantly decreased the number of PSCs (Figure 2B). Among them, the 
1 knockdown of 52 uPRA genes decreased the number of HDFs too (Figure $2 \mathrm{C}$ ). This

2 result indicates that uPRA genes have important effects on the cellular maintenance

3 of HDFs as well. Additionally, we identified 20 PSC-uPRA genes and 1 HDF-uPRA

4 gene that are each specifically necessary for the survival of the respective cells

5 (Figure 2C; images of the knockdown of these 21 genes are shown in Figure S2). A

6 GO analysis showed that the 20 PSC-uPRA genes code for components of

7 ribonucleoprotein and protein-containing complexes and have RNA- and nucleic

8 acid-binding properties (Figure 2D, E). These data suggest that the 20 PSC-uPRA

9 genes are essential for the survival of PSCs and synergistically maintain PSCs via

10 heterocyclic compound binding properties.

12 Upregulated protein levels of the $\mathbf{2 0}$ essential uPRA genes are PSC-specific

13 We examined if the 20 PSC-uPRA genes are regulated post-transcriptionally only in

14 PSCs. We selected another seven differentiated primary cell lines representing the

15 three germ layers to compare with the PSCs, which are undifferentiated. In total,

16 mesoderm-derived cells included adipose tissue-derived mesenchymal stem cells

17 (HAdMSC) and two HDF lines (HDF-1 and 2), endoderm-derived cells included

18 normal human bronchial epithelial cells (NHBEC) and human prostate epithelial cells

19 (PrEC), and ectoderm-derived cells included normal human epidermal keratinocytes

20 (NHEK) and human neural progenitor cells derived from H9 (NPC H9). Quantitative

21 reverse transcription PCR (qRT-PCR) and immunoblotting were performed on the

22 mRNA and proteins, respectively, and the gene expressions relative to HDF-1 were

23 compared (all blotting images and graphs are shown in Figure S3A, C). RSL1D1,

24 one of the 20 essential PSC-uPRA genes, showed a more than 50-fold increase in

25 its protein expression only in PSCs, whereas its mRNA expression was equal among

26 all cell types (Figure 3A, B). Overall, we confirmed that the protein expressions of 18

27 PSC-uPRA genes (RBM22 and SF3B3 were the exceptions) were increased more

28 than two-fold compared with their mRNA expressions in PSCs (Figure 3C). On the

29 other hand, the fold-change between protein and mRNA expressions was around 
1 one or less in differentiated cells for 17 PSC-uRPO genes (IMP4, NCBP2, and

2 BUD31 were the exceptions), suggesting the post-transcriptional regulation

3 mechanism is different between PSCs and differentiated cells (Figure 3D). RSL1D1,

4 SF3B4, RBM22, and SF3B3 especially showed protein to mRNA expression ratios

5 less than one in differentiated cells, indicating that the protein expressions of these

6 genes are usually suppressed in cell types other than PSCs. As for AFP, the one

7 HDF-uPRA gene, we confirmed a specific increase in the protein expression in

8 mesoderm-derived cells (Figure S3B). Following these observations, we concluded

9 that the 20 PSC-uPRA genes are post-transcriptionally regulated specifically in

10 PSCs.

12 HSPA8, EIF3D, and NCBP2 protein expressions are controlled at the ubiquitin13 dependent degradation stage

14 Next, we analyzed individual post-transcriptional regulation stages to identify the 15 regulation mechanism of the protein expressions of the 20 essential PSC-uPRA 16 genes (Figure 4A). After transcription, mRNAs are transported from the nucleus to 17 the cytoplasm, where ribosomes bind to translate them. Eventually, the proteins are 18 degraded by the ubiquitin-proteasome system and/or lysosomes. At first, we 19 analyzed the protein degradation efficiency using MG-132 (proteasome inhibitor) 20 and Bafilomycin A1 and Wortmannin (lysosome inhibitors) as controls for the assays 21 (Figure S4A-D). These inhibitors should recover the PSC-uPRA protein expression 22 in HDFs if the proteins are downregulated by fast degradation. We found that the 23 protein expressions of HSPA8, EIF3D, NCBP2, and IMP4 were steadily increased 24 more than two-fold after exposure to proteasome inhibitor for up to 8 hours (Figure 25 4B, S4E). We did the same assay for up to 24 hours and confirmed that the protein 26 expressions of HSPA8, EIF3D, and NCBP2 were controlled by fast degradation via 27 the proteasome system in HDFs (Figure 4C). The lysosome inhibitor assay (Figure 28 4D, S4F) showed high protein expressions of NCBP2 and RSL1D1 for treatment up 29 to 8 hours but not 24 hours (Figure 4E), suggesting no PSC-uPRA genes were 
1 regulated by lysosome-dependent degradation. Overall, these data indicate that the

2 protein expressions of three PSC-UPRA genes, HSPA8, EIF3D and NCBP2, are

3 regulated at the ubiquitin dependent degradation stage. increased in PSCs

7 Next, we analyzed the subcellular localization of the mRNAs of the 20 essential PSC-

8 UPRA genes to examine their intracellular abundance between different cell types.

9 We used 18S rRNA and MALAT1 IncRNA, respectively, as cytosolic and nuclear

10 RNA controls in the subcellular fractionation experiment (Figure 5A). For cytosolic

11 RNA, 94.3\% and 73.3\% of 18S rRNA were observed in PSCs and HDFs, respectively,

12 and for nuclear RNA, 99.5\% and 99.8\% of MALAT1 IncRNA were observed,

13 validating the subcellular fractionation experiment. Then we analyzed the cytosolic

14 RNA percentage of the 20 essential PSC-uPRA genes by qRT-PCR (Figure 5B). The

15 cytosolic RNA percentage of 18 PSC-uPRA genes (IMP4 and CPSF6 were the

16 exceptions) were increased at least more than two-fold in PSCs. Conversely, the

17 nuclear RNA percentage of PSC-UPRA genes was increased in HDFs (Figure 5C).

18 These results indicated that the mRNAs of the 18 PSC-uPRA genes were retained

19 in the nucleus in HDFs.

20 RNA splicing is known to promote mRNA-export to the cytoplasm, because export

21 factors can be recruited by the spliceosome to mature RNA (Palazzo and Lee, 2018).

22 However, PSCs and differentiated cells do not express different spliced isoforms of

23 the 20 PSC-uPRA genes (Han et al., 2013). We then checked the protein

24 expressions of RNA export factors. We found more than a four-fold increase in the

25 protein expressions of NXT1 and NXF1, two mRNA export receptor heterodimers, in

26 PSCs and NPC 1418E1 compared to the other differentiated cells (Figure 5D, S5A,

27 B). These data indicated that most PSC-uPRA mRNA was retained in the nucleus of

28 HDFs, but the increased NXT1 and NXF1 protein expressions may accelerate mRNA

29 transport to the cytoplasm in PSCs. 
1 Next, to investigate the translation efficiency, we extracted mRNAs in the monosome,

2 light polysome, and heavy polysome fractions of PSCs and HDFs using sucrose

3 fractionation. We quantified and compared the PSC-uPRA mRNA in each fraction

4 between PSCs and HDFs (Figure 5E). Except for NIFK and RSL1D1, no PSC-uPRA

5 mRNA was enriched in the heavy polysome fraction of PSCs (Figure 5F). This result

6 suggests that the translation efficiency of most PSC-uPRA mRNAs cannot explain

7 the increased protein levels in PSCs.

8 Among PSC-UPRA mRNAs, SRRT mRNA was the most associated with heavy

9 polysomes in HDFs despite the inhibited protein expression (Figure 5F). Since a

10 lower cytosolic RNA percentage of endogenous SRRT was observed in HDFs

11 (around 10\%; Figure 5B), we next analyzed whether its translation occurs if the

12 cytoplasmic RNA content is increased. We constructed full-length FLAG-tagged

13 SRRT mRNA and one day after the mRNA transfection confirmed the translation of

14 FLAG-SRRT protein from the transfected mRNA by immunoblotting (Figure 5G). In

15 summary, these results indicate that there exists an inhibitory mechanism in HDFs

16 for transporting PSC-UPRA mRNAs to the cytoplasm and for the translation of PSC-

17 UPRA genes even after mRNA binds to ribosomes.

\section{DISCUSSION}

20 Despite their ubiquitous effects, there is comparatively little understanding about 21 post-transcriptional regulatory mechanisms compared with transcriptional regulatory 22 mechanisms in PSCs. Here, we revealed that 20 post-transcriptionally regulated 23 genes (i.e. UPRA genes) are essential for the maintenance of PSCs. These genes 24 had upregulated protein levels without any major positive upregulation in mRNA 25 levels in PSCs (Figure 3C). The regulatory processes of these 20 essential PSC26 uPRA genes are illustrated in Figure 6. In HDFs, 18 PSC-uPRA mRNAs were highly 27 retained in the nucleus (Figure 5C). Among them, the translation efficiency of RSL1D1 and NIFK was upregulated in PSCs (Figure 5F), and the proteins of HSPA8, 
1 stage (Figure 4B, C). Also, we found that the translation efficiency of 18 uPRA genes

2 was comparable between PSCs and HDFs by polysome profiling (Figure 5F), suggesting several inhibitory effects for UPRA mRNA transportation to the cytoplasm and for translation after ribosome binding in differentiated cells.

5 To identify the inhibitory effect, we picked up one UPRA gene, SRRT, for further analysis. The percentage of cytoplasmic SRRT mRNA was 3-fold less in HDFs (Figure 5B), and the mRNA itself was more associated with heavy polysomes in

8 HDFs than in PSCs (Figure 5F). Furthermore, exogenous cytosolic SRRT mRNA was translated in HDFs (Figure 5G), suggesting the cytoplasmic mRNA level is key for proper protein expression. Another possible mechanism for translation inhibition

11 in differentiated cells is ribosome pausing, in which the mRNA binds to ribosomes

12 that are not active or select non-canonical start codons (Chandrasekaran et al.,

13 2019; Darnell et al., 2018; Tresenrider et al., 2021). A third possibility is that aberrant

14 proteins were degraded immediately after the production by a quality control 15 pathway of the mRNA and ribosome (D'Orazio and Green, 2021). More study is

16 needed to clarify which mechanism applies to which gene.

17 All identified 20 PSC-uPRA proteins in the present study are involved in a wide range 18 of cellular functions, from transcription to post-translation. For transcription-related 19 functions, 10 PSC-uPRA genes have polymerase-, splicing- and mRNA maturation20 related functions: POLD2 is a component of the DNA polymerase complex; SF3B3, 21 SF3B4, and RBM22 are components of the splicing factor; and PUF60, BUD31 22 SAP18, CPSF6, U2AF2, and SRRT are required for the splicing of pre-mRNA, with 23 SRRT especially known as "a molecular guardian of the pluripotent cell state" by 24 facilitating proper splicing in PSCs (Kainov and Makeyev, 2020). For translation25 related functions, 8 PSC-uPRA genes are involved in functions of the ribosome 26 complex: NCBP2 and EIF3D are mRNA cap binding proteins; EIF3D is necessary 27 for specialized translation initiation factors (Lee et al., 2016); RPL7L1 is a putative 28 component of the ribosome complex; and RSL1D1, NIFK, NOL10, IMP4, and TSR1 29 are required for rRNA processing. For post-translational functions, CCT5 and HSPA8 30 are components of the chaperone complex, which helps newly synthesized proteins 31 properly fold. Our observation that these genes are post-transcriptionally regulated 
1 is particularly interesting, because they all function as regulators post-transcription.

2 Thus, they might use feedback in their regulation to maintain pluripotency.

3 In conclusion, our study revealed that the protein expressions of 228 genes are post-

4 transcriptionally regulated in PSCs. The protein levels of 20 of these genes are

5 specifically increased in PSCs, and the proteins themselves are essential for PSC

6 maintenance. Finally, different mechanisms regulate the gene expressions between

7 cell types.

$9 \quad$ Limitation of the study

10 We focused on post-transcriptionally regulated genes essential for the maintenance

11 of PSCs. Although we showed critical regulatory steps for controlling uPRA protein

12 levels, a wide variety of regulatory mechanisms seem to be involved. Recent studies

13 have shown that the secondary structure of mRNA is important for regulating protein

14 expression by changing the interaction with the expansion segment of ribosomal

15 RNA (Leppek et al., 2020) and changing the mRNA half-life (Mauger et al., 2019).

16 Future studies are needed to determine if secondary structures affect the translation

17 of the 20 PSC-uPRA genes. 


\section{$1 \quad$ Figure Legends}

Iwasaki et al., Figure 1

A

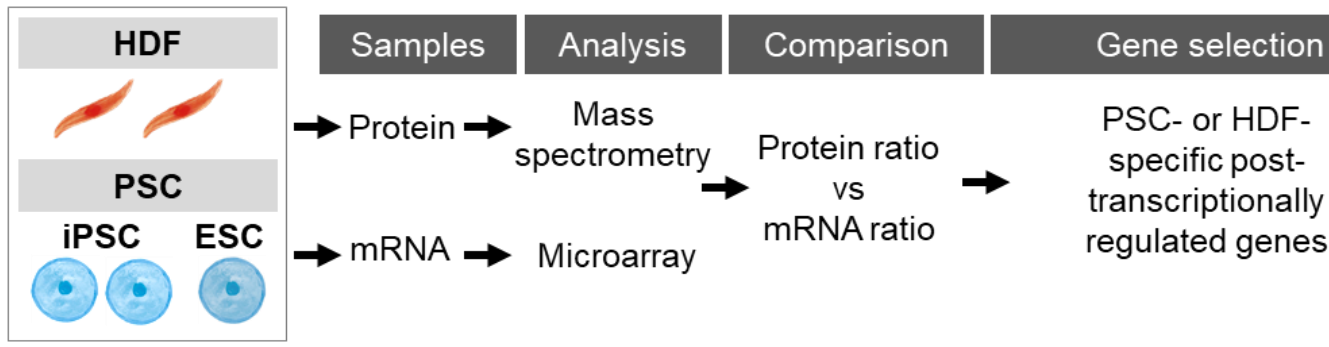

B

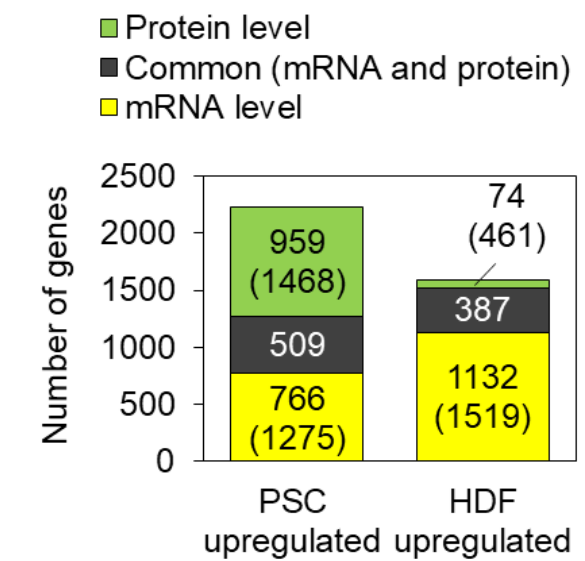

D

\begin{tabular}{|c|c|c|c|c|c|}
\hline & Cell & \# Genes & GO (molecular function) & p-value & Matches \\
\hline \multirow{4}{*}{$\begin{array}{l}\text { Protein upregulated } \\
\text { mRNA no change } \\
(p<0.05) \\
\text { (uPRA genes) }\end{array}$} & \multirow{3}{*}{ PSC } & \multirow{3}{*}{228} & RNA binding & $7.12 \mathrm{E}-97$ & 146 \\
\hline & & & nucleic acid binding & 6.63E-55 & 153 \\
\hline & & & structural constituent of ribosome & 2.96E-50 & 47 \\
\hline & HDF & 8 & no enrichment & -- & -- \\
\hline \multirow{3}{*}{$\begin{array}{l}\text { mRNA upregulated } \\
\text { Protein no change } \\
(p<0.05)\end{array}$} & PSC & 10 & transmembrane transporter activity & 0.03 & 4 \\
\hline & \multirow{2}{*}{ HDF } & \multirow{2}{*}{56} & cadherin binding & 0.01 & 8 \\
\hline & & & cell adhesion molecule binding & 0.01 & 10 \\
\hline
\end{tabular}

C

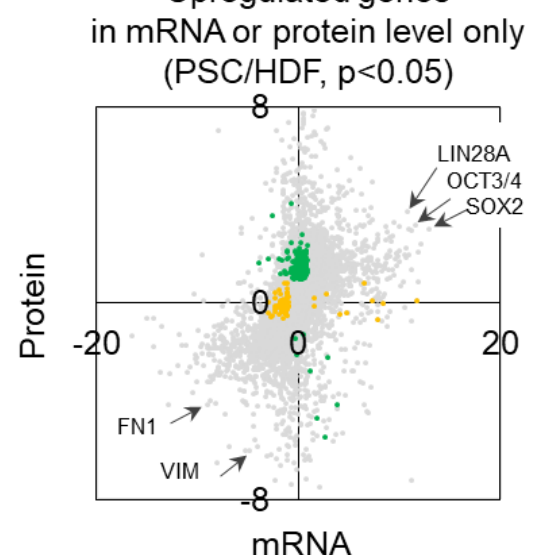

Figure 1 | 228 and 8 genes were respectively identified as posttranscriptionally regulated genes with independent protein upregulation in PSCs and HDFs

A. Workflow for identifying post-transcriptionally regulated genes using mass spectrometry (MS) for proteins and microarray for mRNA. Two iPSC lines (iPSC- 
1, 201B7; and iPSC-2, 1418E1), one ESC line (H9), and two HDF lines (HDF-1, 2 HDF1388; and HDF-2, Tig120) were used.

3 B. Number of commonly upregulated genes by protein levels only, mRNA levels only, and both levels in PSCs and HDFs. Numbers in parentheses show the total numbers.

C. The mRNA to protein expression ratio in PSCs and HDFs for each gene. Gray, yellow, and green indicate all genes, those with only upregulated mRNA, and those with only upregulated protein levels, respectively $(p<0.05$, two-sample unpaired $t$ test). $\log _{2}$ scale. Comparisons of the mRNA and protein ratios between all PSC and HDF lines are shown in Figure S1. The total number of genes quantified was 6404. All plotted data are shown in Table S1. See "Transomics data analysis" in Methods for details.

D. Number of genes and the $\mathrm{GO}$ analysis of molecular functions for genes that were significantly upregulated only at the protein or mRNA level in C. 
A Knockdown of post-transcriptionally regulated

Iwasaki et al., Figure 2 genes in PSC and HDF (156 uPRA genes)
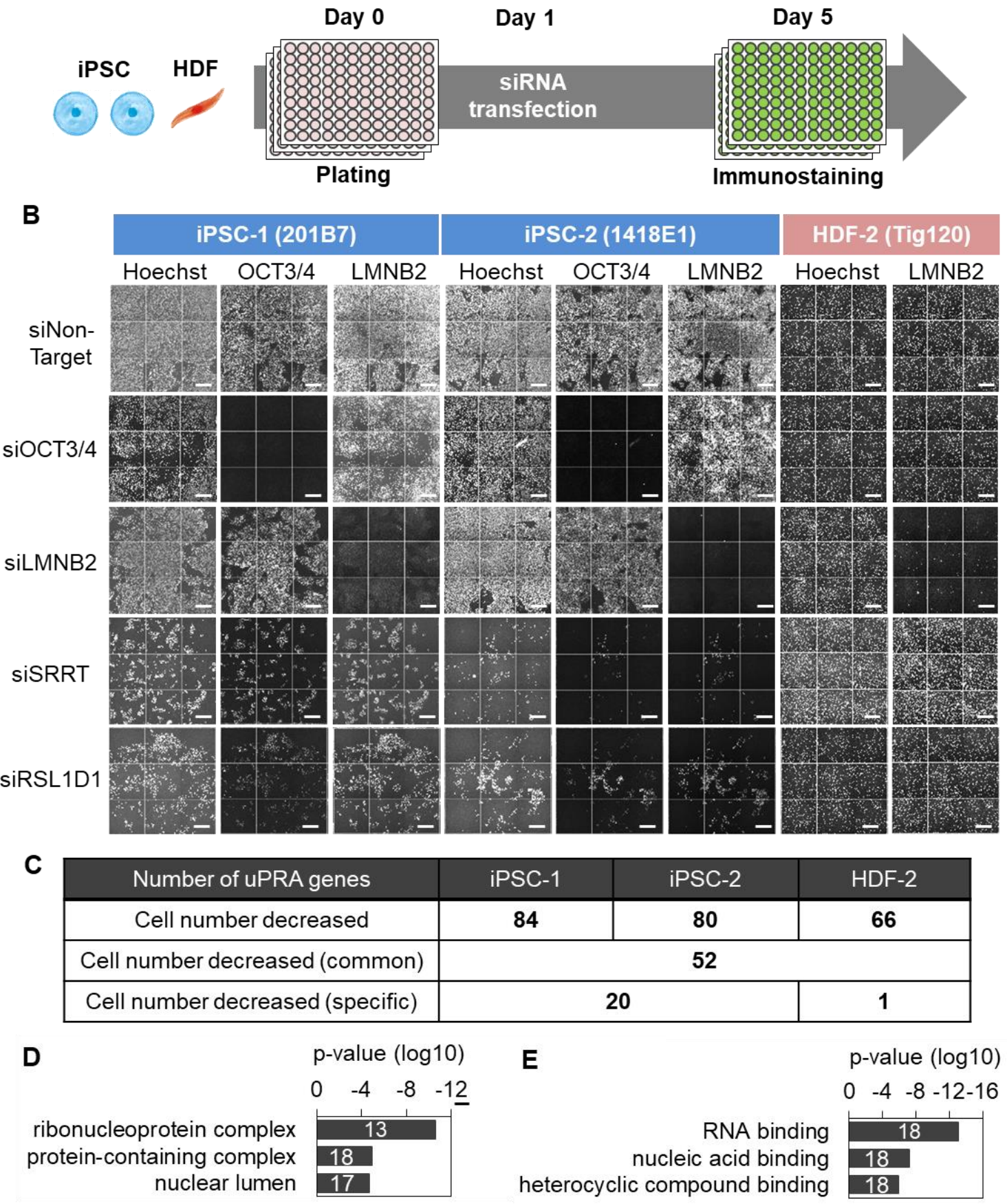

Figure 2 | 20 uPRA genes in PSCs and 1 uPRA gene in HDFs were related to

3 cell survival

4 A. Workflow of the knockdown experiment by siRNA for uPRA genes in PSCs and 
HDFs (156 uPRA genes in total). iPSC-1 (201B7), iPSC-2 (1418E1), and HDF2 (Tig120) were used for the assay. See "siRNA screening" in Methods for details. The knockdown targets are shown in Table S2, and the immunoassay cell percentage and observed number of cells are shown in Table S3.

D. GO analysis of cellular component for the 20 PSC-uPRA genes in C.

E. GO analysis of molecular function for the 20 PSC-uPRA genes in C. 
A

RSL1D1

$\beta$-ACTIN
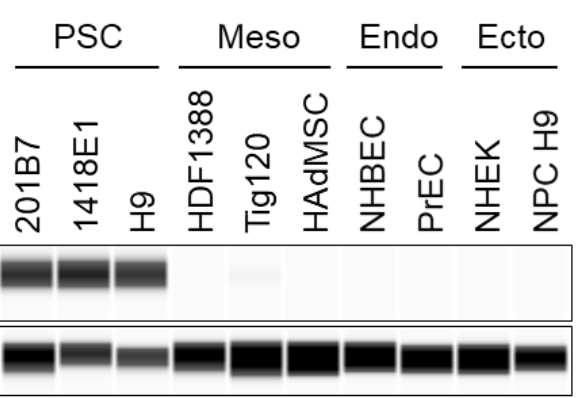

B

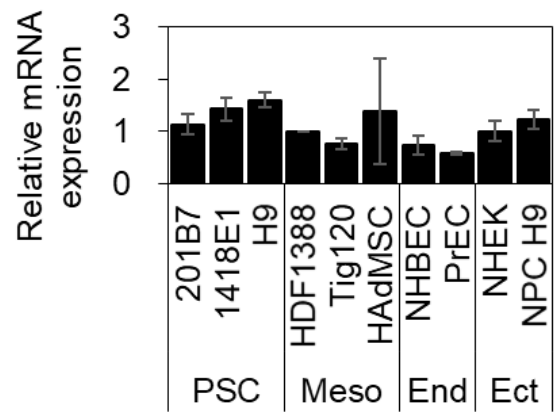

C

D
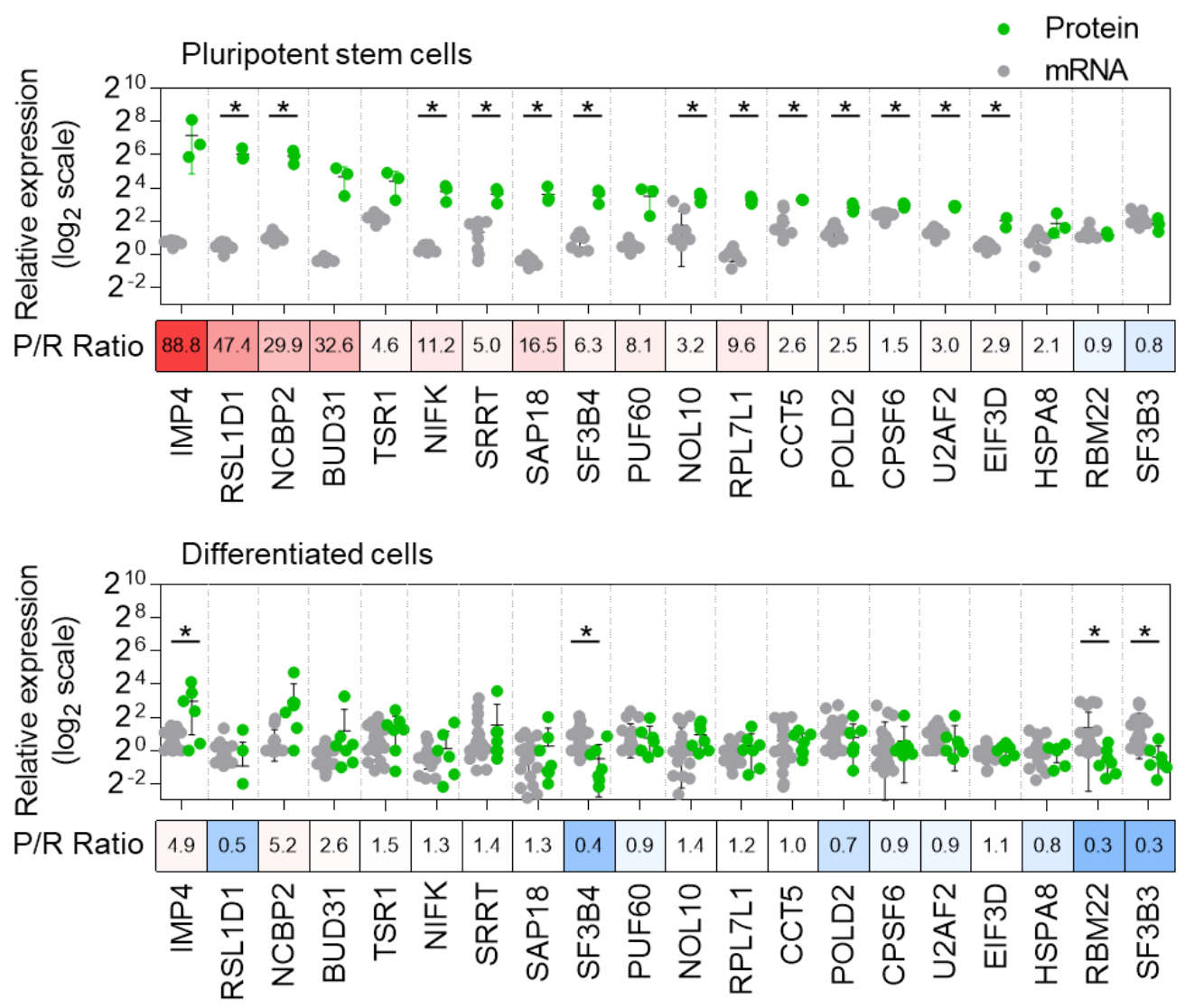

Figure 3 | 20 uPRA genes were post-transcriptionally regulated only in PSCs

A. A representative image of the immunoblotting for one PSC-UPRA gene, RSL1D1, in various cell types. We used three cell lines for PSCs, three cell lines for mesoderm-derived cells, two cell lines for endoderm-derived cells, and two cell lines for ectoderm-derived cells for the gene expression analysis. 
1 Immunoblotting images of all 20 essential PSC-uPRA genes are shown in Figure 2 S3A.

B. Gene expression measured by qRT-PCR was normalized by GAPDH. The relative expression ratios were normalized to the result of HDF1388 (HDF-1). Representative results for the RSL1D1 mRNA are shown as the mean \pm S.D. Biological triplicates for the mRNA quantification. Results for all essential PSCUPRA genes are shown in Figure S3C.

C. Relative gene expressions of mRNA and protein levels in PSCs for the 20 essential PSC-uPRA genes normalized to HDF1388 (HDF-1). A heatmap of the protein to mRNA expression ratio (P/R ratio) is shown above each gene name.

$11{ }^{*} p<0.05$, two-sample unpaired t test.

12 D. Relative mRNA and protein levels in differentiated cells for the 20 essential PSCuPRA genes normalized to HDF1388 (HDF-1). A heatmap of the P/R ratio is shown above each gene name. ${ }^{*} p<0.05$, two-sample unpaired t test. 
A

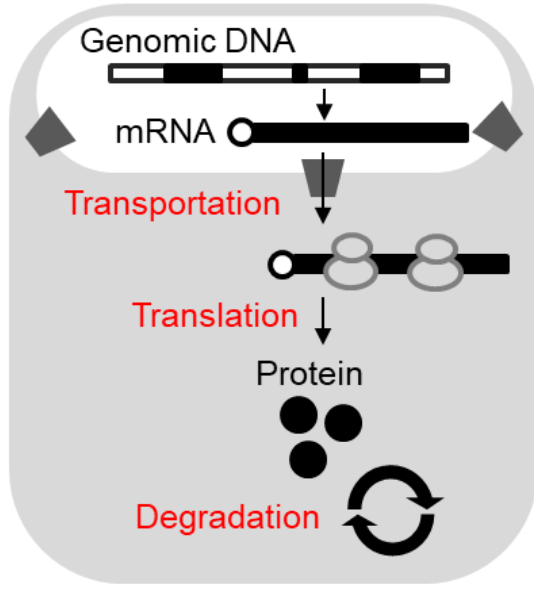

B

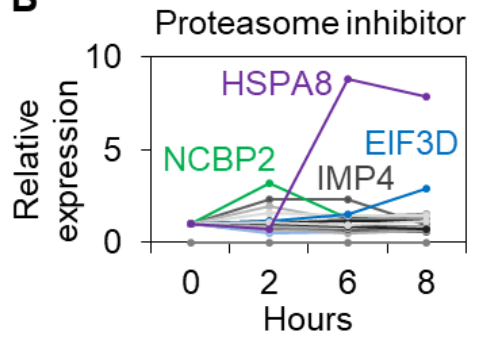

C

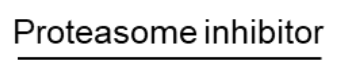

Iwasaki et al., Figure 4

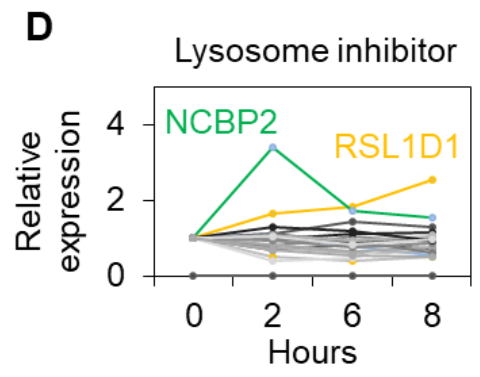

E

Lysosome inhibitor $\frac{\text { HDF }}{\text { Oh } 2 \mathrm{~h} 24 \mathrm{~h}} \frac{\mathrm{iPSC}}{\text { Oh } 1 \mathrm{~h}}$

NCBP2

RSL1D1

$\beta-A C T I N$

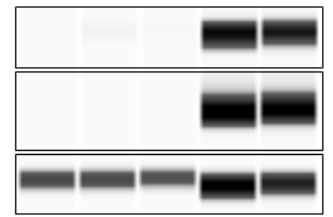

Figure 4 | HSPA8, EIF3D, and NCBP2 protein expressions are controlled at the ubiquitin-dependent degradation stage

A. Overview of the known regulation stages for gene expression after transcription. to 8 hours, and the expression levels of the 20 essential PSC-uPRA genes were measured by immunoblotting. The relative expression of each protein compared to pre-inhibition is shown. All immunoblotting images are shown in Figure S4A.

C. HDF-2 (Tig120) and iPSC-1 (201B7) were treated with $20 \mu \mathrm{M}$ MG-132 for up to 24 hours, and the protein expressions for HSPA8, EIF3D, NCBP2, and IMP4 were measured by immunoblotting.

D. HDF-2 (Tig120) was treated with lysosome inhibitors (250 nM Bafilomycin A1 and $500 \mathrm{nM}$ Wortmannin) for up to 8 hours, and the protein expression of the 20 essential PSC-uPRA genes were measured by immunoblotting. The relative expression of each protein to pre-inhibition is shown. All immunoblotting images 

are shown in Figure S4B.

2 E. HDF-2 (Tig120) and iPSC-1 (201B7) were treated with $250 \mathrm{nM}$ Bafilomycin and $3500 \mathrm{nM}$ Wortmannin for up to 24 hours, and the protein expressions of NCBP2 $4 \quad$ and RSL1D1 were measured by immunoblotting. 


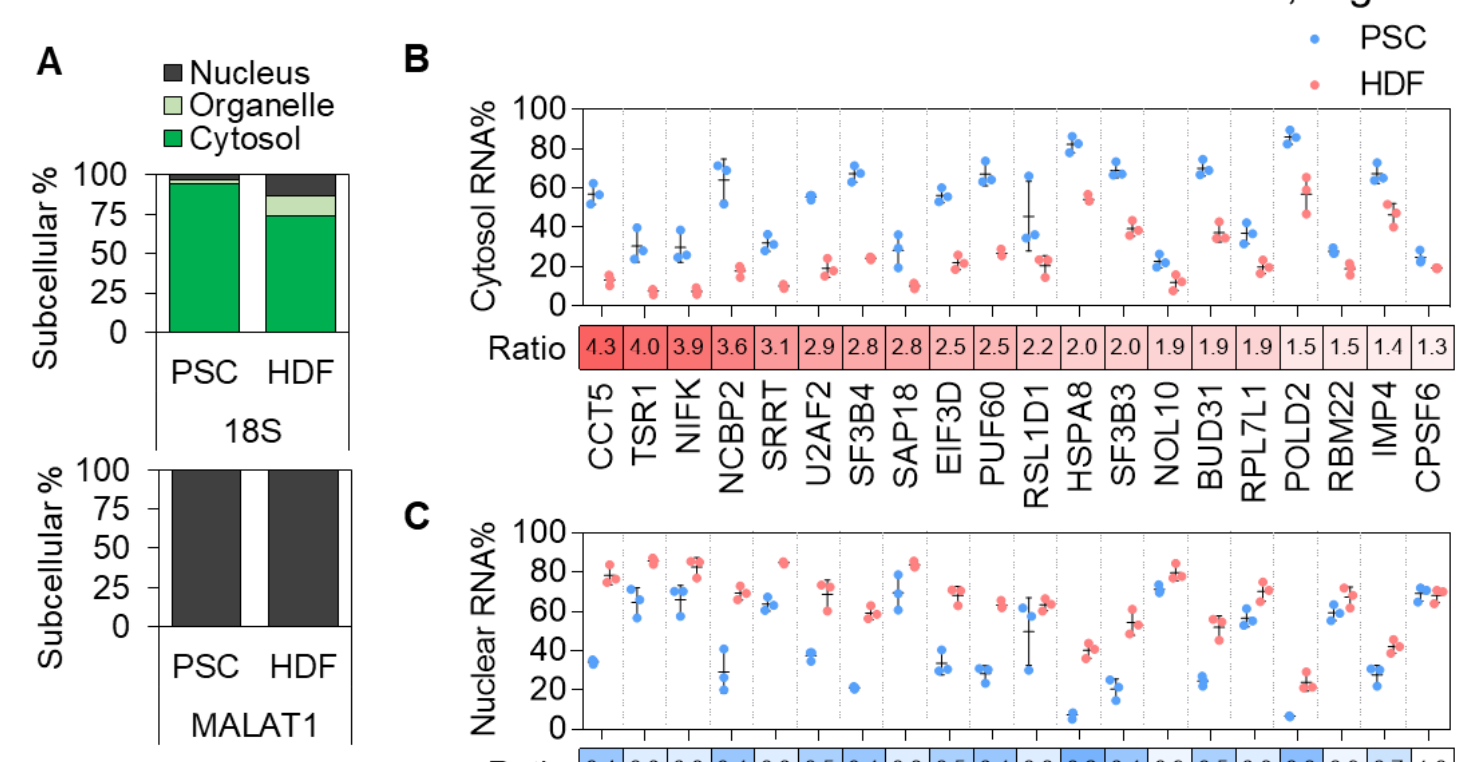

B

Iwasaki et al., Figure 5

D
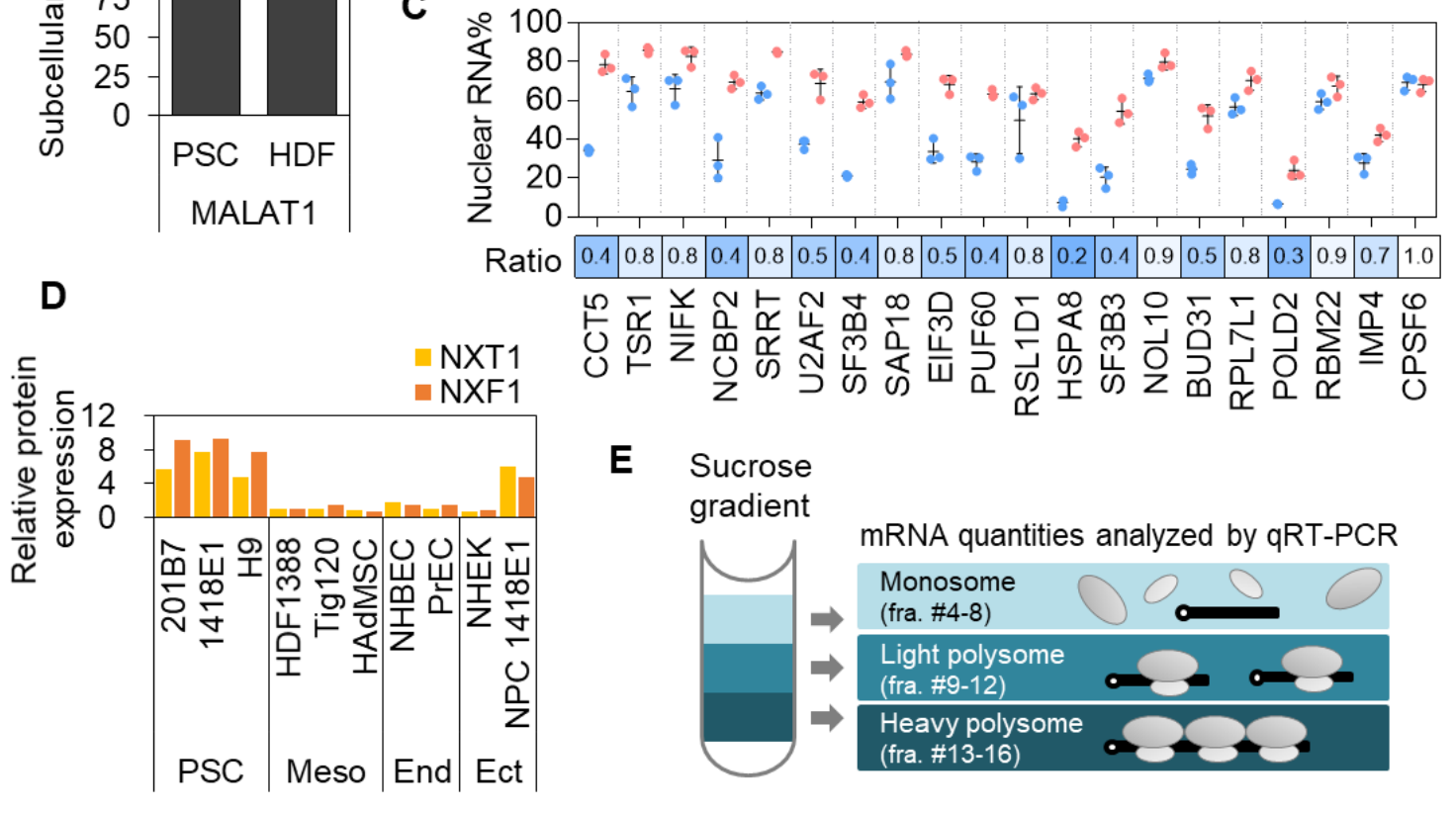

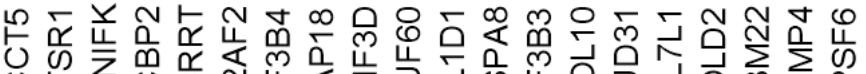

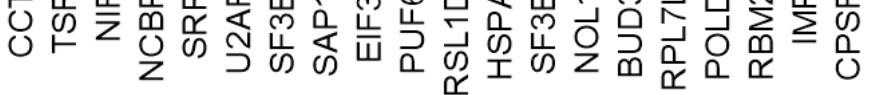

\section{E Sucrose} gradient

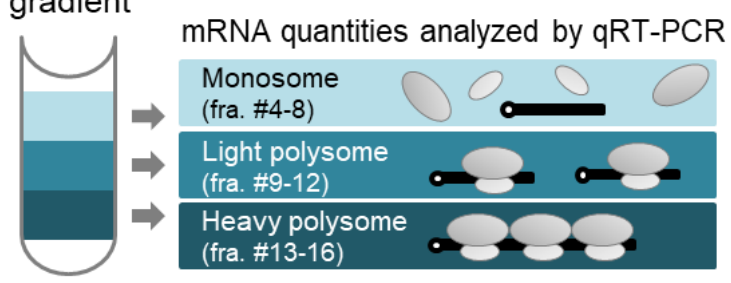

$\mathbf{F}$

\begin{tabular}{ll|l|l|l|l|l|l|l|l|l|l|l|l|l|l|l|l|l|l|l|} 
Mono & 2.8 & 2.2 & 2.1 & 1.9 & 1.4 & 0.8 & 0.5 & 0.4 & 0.4 & 0.2 & 0 & 0 & -0.1 & -0.2 & -0.4 & -0.5 & -0.7 & -0.9 & -1.0 & -2.8 \\
$\square$
\end{tabular}

Light Poly

Heavy Poly

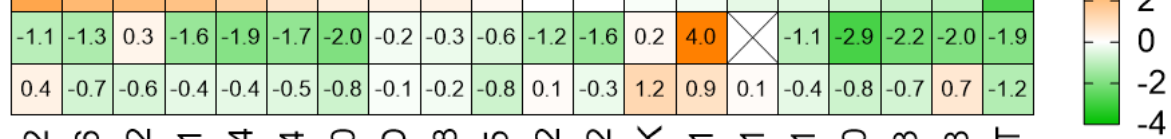

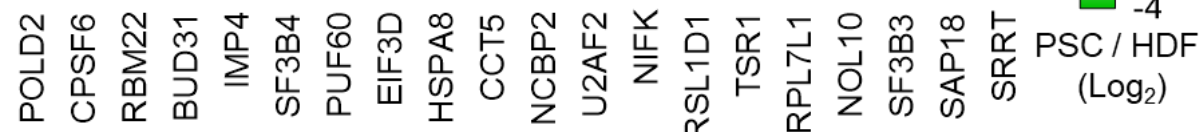

G

mRNA transfection

\begin{tabular}{|c|c|}
\hline & HDF iPSC \\
\hline & $-F L-F L$ \\
\hline FLAG & $=$ \\
\hline SRRT & $=0$ \\
\hline$\beta$-ACTIN & (1) \\
\hline
\end{tabular}


1 Figure 5 | PSC-uPRA mRNAs are retained in the nucleus of HDFs without 2 changing the translation efficiency

A. The percentages of mRNA in the cytoplasm, membrane, and nucleus of iPSC-1 (201B7) and HDF-2 (Tig120) are shown for 18S ribosomal RNA and MALAT1 IncRNA measured by qRT-PCR.

B. The percentage of mRNA in the cytoplasm measured by qRT-PCR for the 20 essential PSC-uPRA genes. The cytoplasm mRNA expression ratio in PSCs to HDFs is shown above each gene name in the heatmap.

C. The percentage of mRNA in the nucleus measured by qRT-PCR for the 20 essential PSC-UPRA genes. The nucleus mRNA expression ratio in PSCs to HDFs is shown above each gene name in the heatmap.

D. The protein-fold change between PSCs and other differentiated cells for NXT1 and NXF1. Protein expressions measured by immunoblotting were normalized by $b-A C T I N$. The relative expression ratios were normalized to the result of HDF1388 (HDF-1).

E. Overview of the analysis of the translation efficiency by the sucrose gradient. The monosome (fraction 4-8), light polysome (fraction 9-12), and heavy polysome (fraction 13-16) were collected to extract RNAs, which were measured by qRT-PCR. See "Monosome and polysome fractionation" in Methods for details.

F. The percentage of mRNAs in monosome (Mono), light polysome (Light Poly), and heavy polysome (Heavy Poly) were analyzed by qRT-PCR. The average mRNA expression ratio in PSCs to HDFs is shown in the heatmap with $\log _{2}$ scale $(n=3)$. Values are normalized by spike RNA and compared with the loading sample before the sucrose gradient.

G. Full-length FLAG-SRRT mRNA was transfected into HDF-2 (Tig120) and iPSC1 (201B7). Immunoblotting assays for FLAG, SRRT, and b-ACTIN are shown. 
Iwasaki et al., Figure 6

Translation efficiency mRNA transport

Protein degradation via proteasome

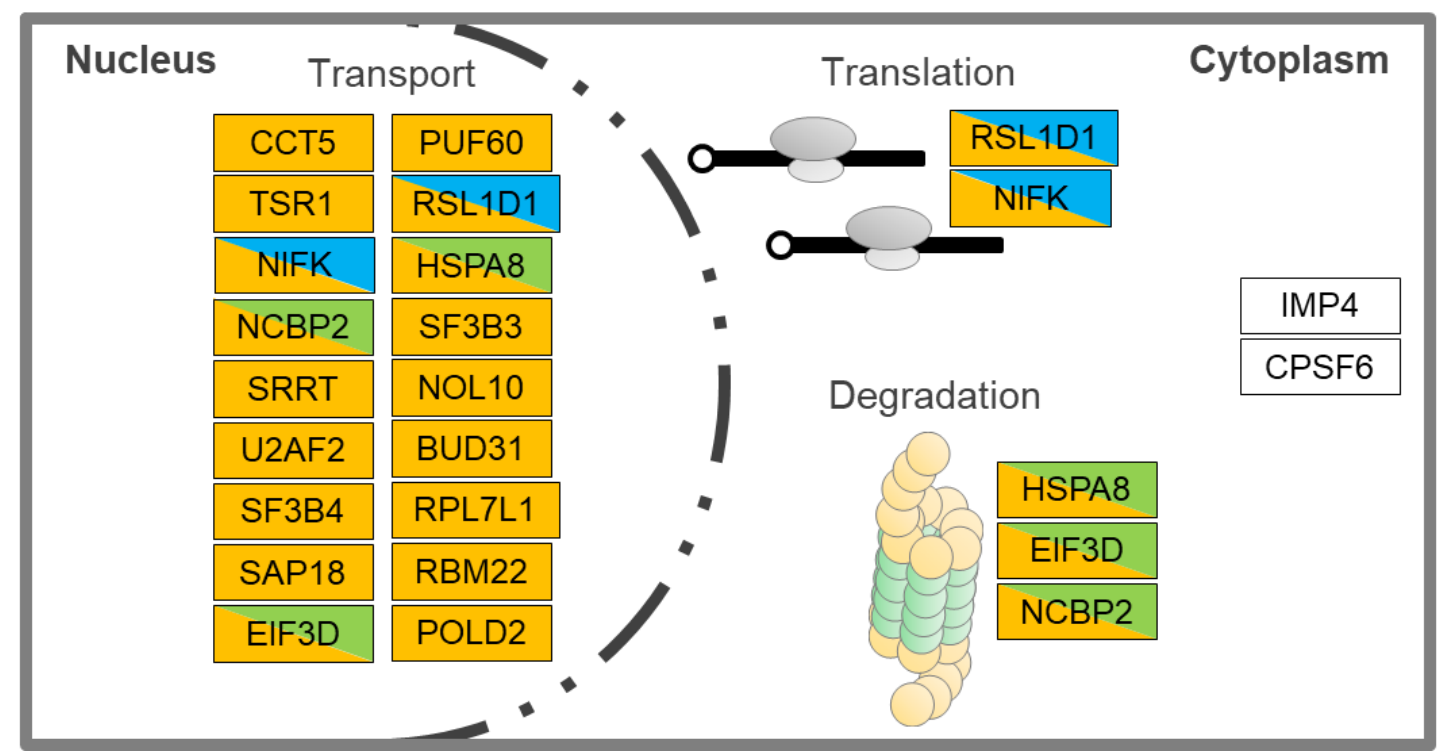

Figure 6 | An illustration of how 20 essential PSC-uPRA genes are regulated in PSCs and differentiated cells

Blue, yellow, and green indicate the translation efficiency, mRNA transport, and protein degradation via the proteasome of the 20 essential PSC-uPRA genes, respectively. More mRNA of 18 uPRA genes was transported from the nucleus to the cytoplasm in PSCs than in HDFs. The proteins of HSPA8, EIF3D, and NCBP2 were quickly degraded in HDFs at the proteasome degradation stage. The translation efficiency of RSL1D1 and NIFK was higher in PSCs compared to HDFs. No regulation was observed for IMP4 or CPSF6. 


\section{Supporting Information}

Iwasaki et al., Supplementary Figure 1

A
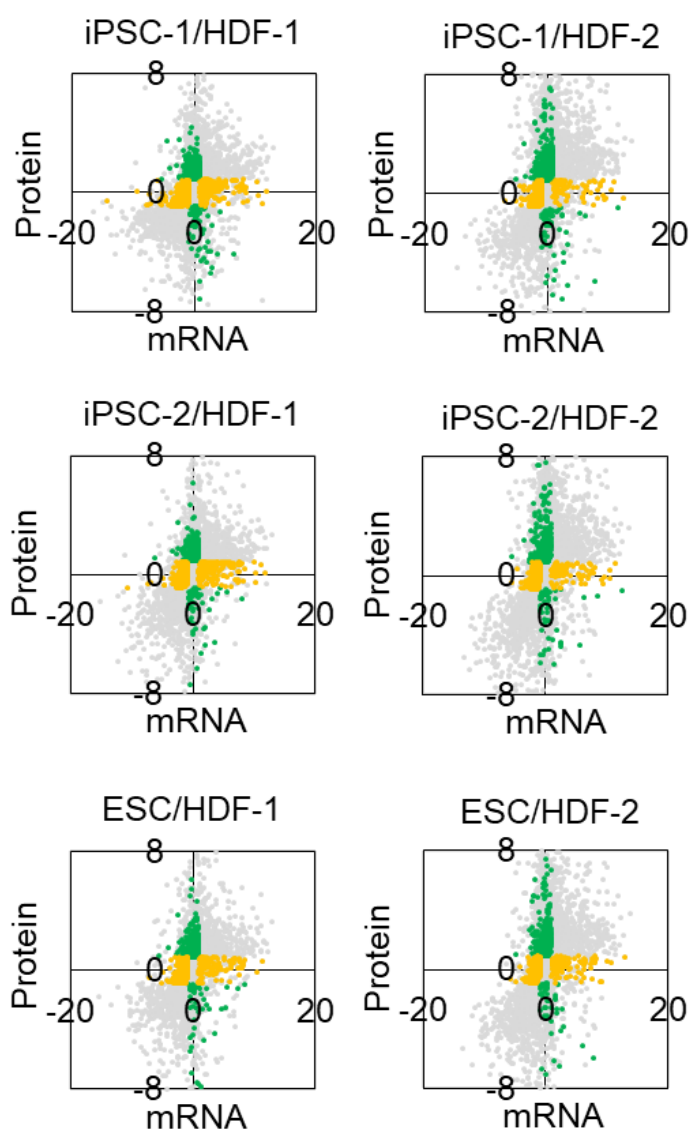

B
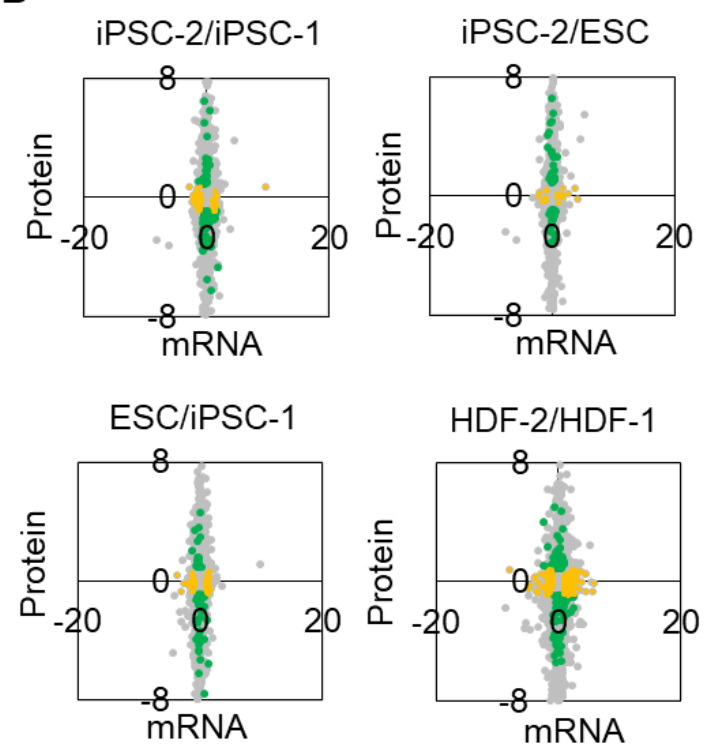

Figure S1 | Comparison of the mRNA to protein ratio between cell lines

4 A. The mRNA to protein ratio for each gene between different PSC and HDF

5 lines. Genes with significantly upregulated mRNA and protein levels only are shown

6 in yellow and green, respectively ( $p<0.05$, two sample paired t test).

7 B. The mRNA to protein ratio for each gene between the same cell type. Genes

8 with significantly upregulated mRNA and protein levels only are shown in yellow and

9 green, respectively ( $p<0.05$, two sample paired t test). 
bioRxiv preprint doi: https://doi.org/10.1101/2021.10.18.462967; this version posted October 19, 2021. The copyright holder for this preprint

(which was not certified by peer review) is the author/funder, who has granted bioRxiv a license to display the preprint in perpetuity. It is made available under aCC-BY-NC-ND 4.0 International license.

Iwasaki et al., Supplementary Figure 2

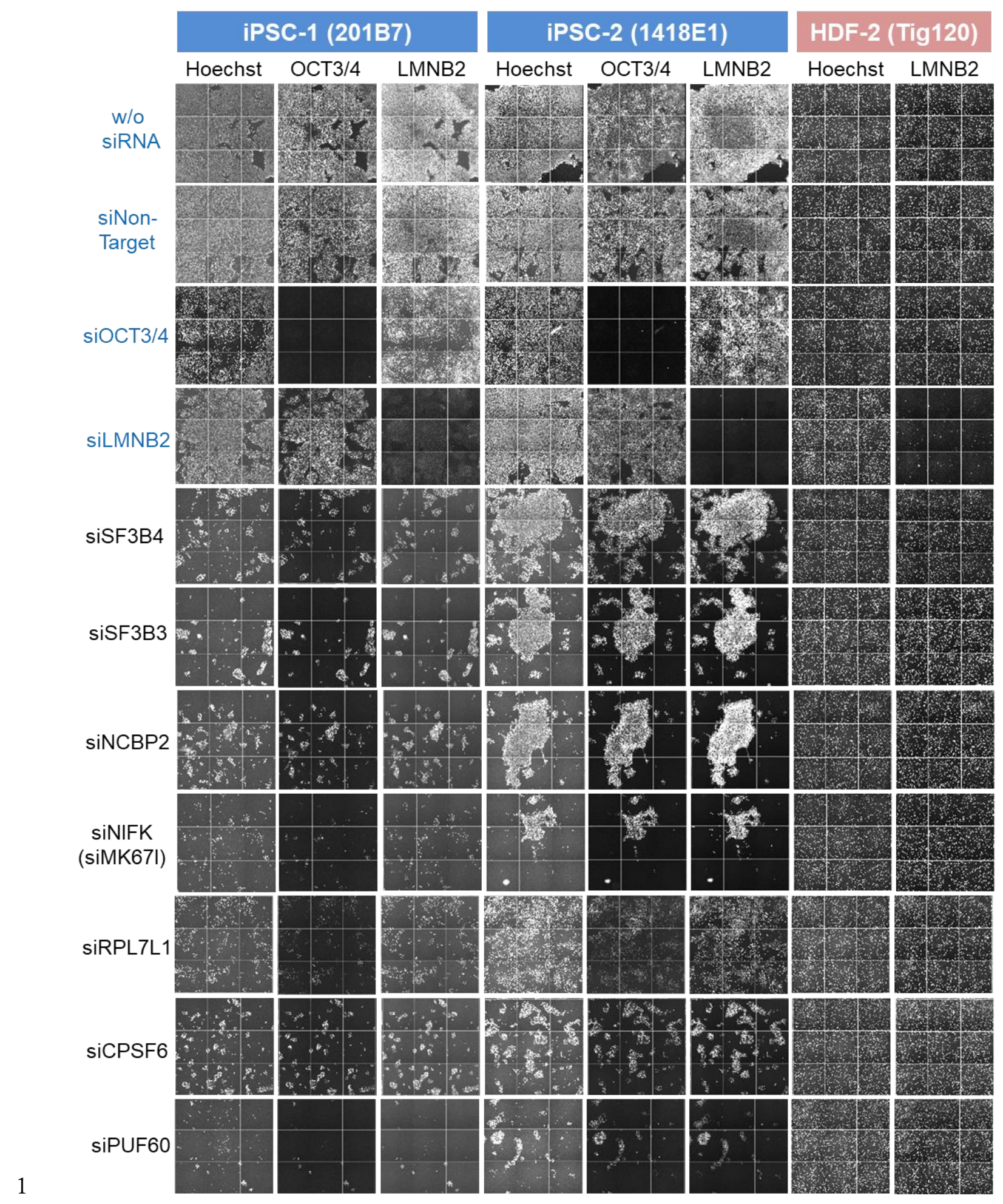


bioRxiv preprint doi: https://doi.org/10.1101/2021.10.18.462967; this version posted October 19,2021 . The copyright holder for this preprint

(which was not certified by peer review) is the author/funder, who has granted bioRxiv a license to display the preprint in perpetuity. It is made available under aCC-BY-NC-ND 4.0 International license.

Iwasaki et al., Supplementary Figure 2

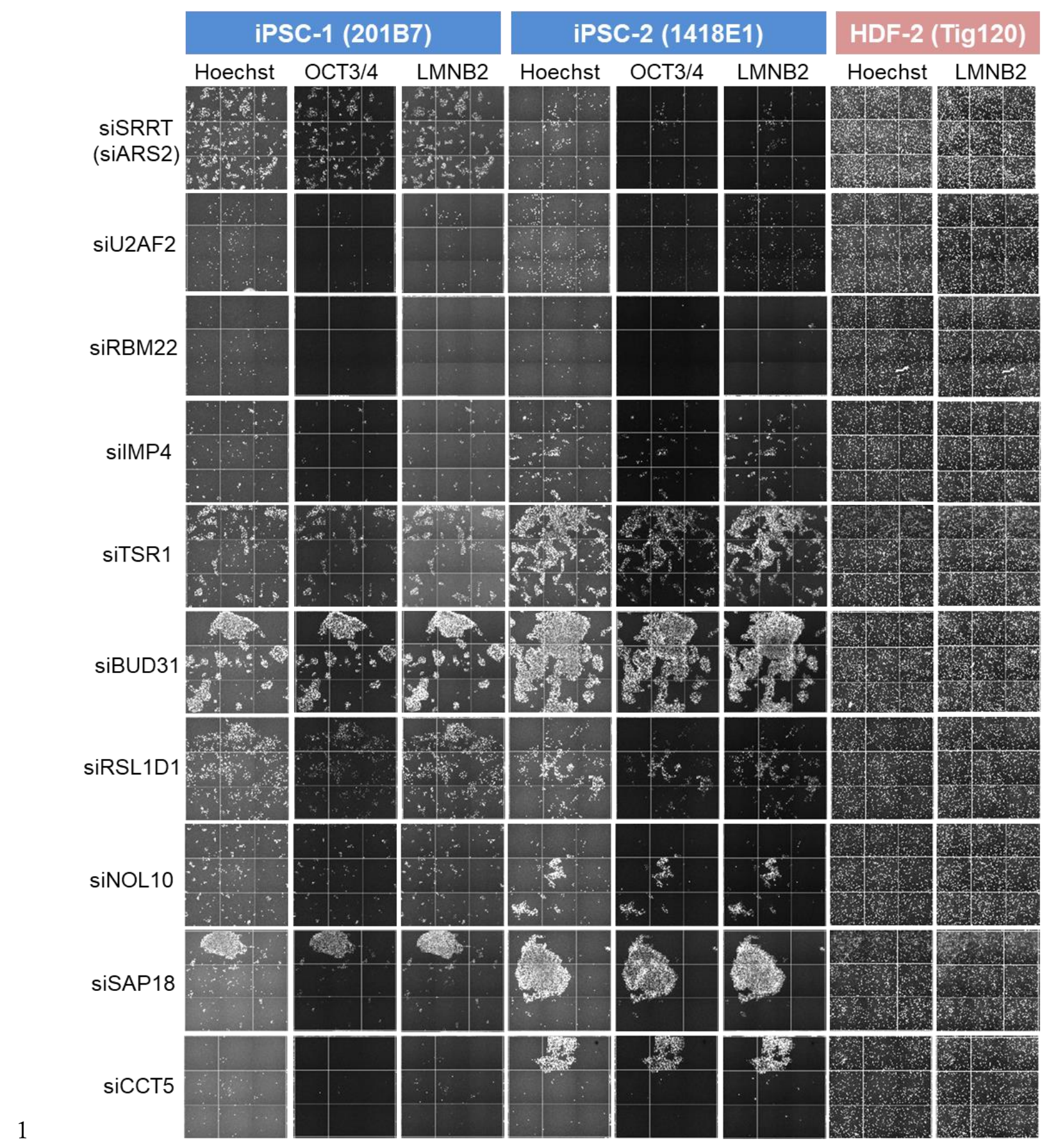


Iwasaki et al., Supplementary Figure 2

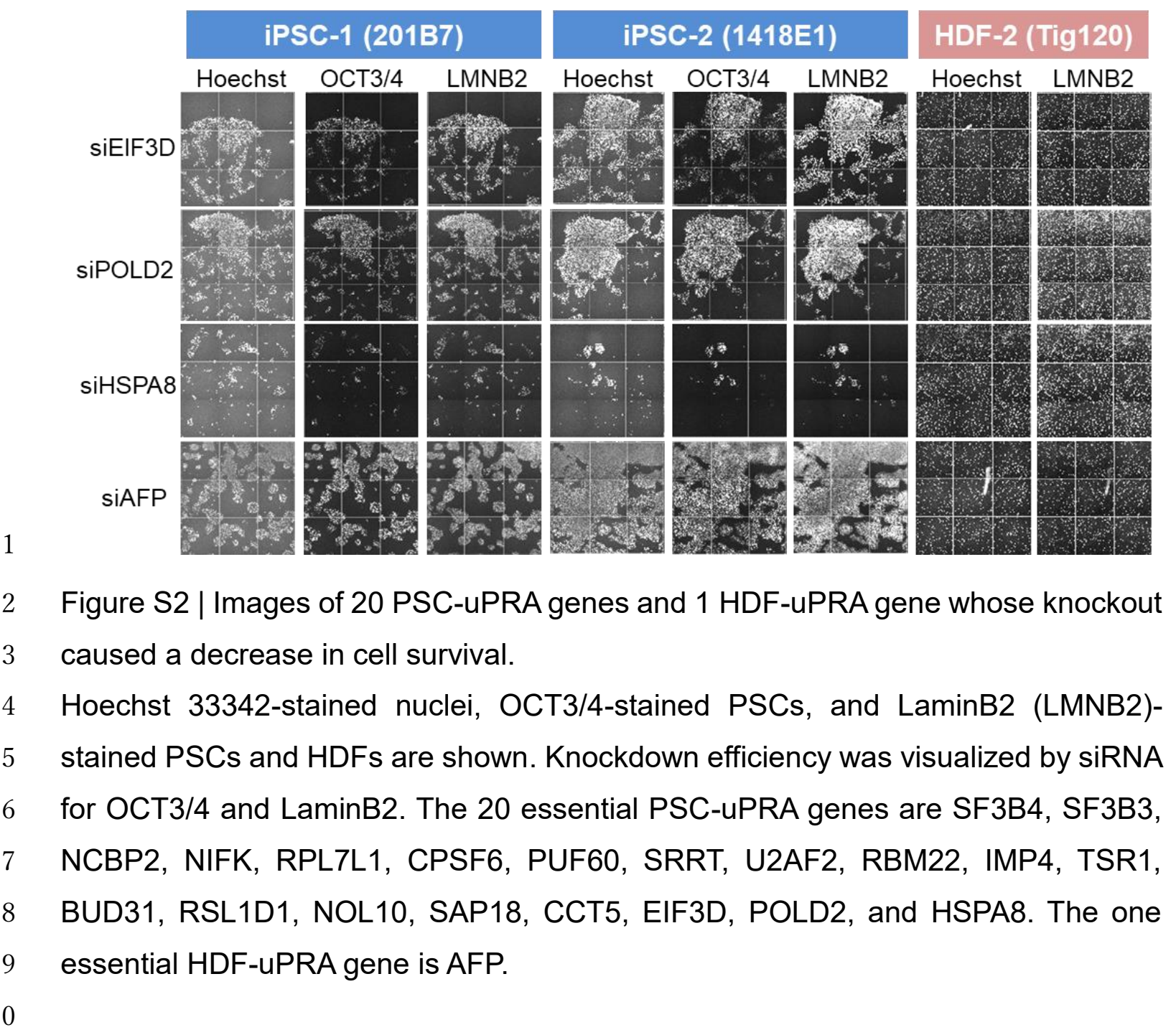


bioRxiv preprint doi: https:/doi.org/10.1101/2021.10.18.462967: this version posted October 192021. The copyright holder for this preprint (which was not certified by peer review) is the author/funder, who has granted bioRxiv a license to display the preprint in perpetuity. It is made available under aCC-BY-NC-ND 4.0 International license.

Iwasaki et al., Supplementary Figure 3
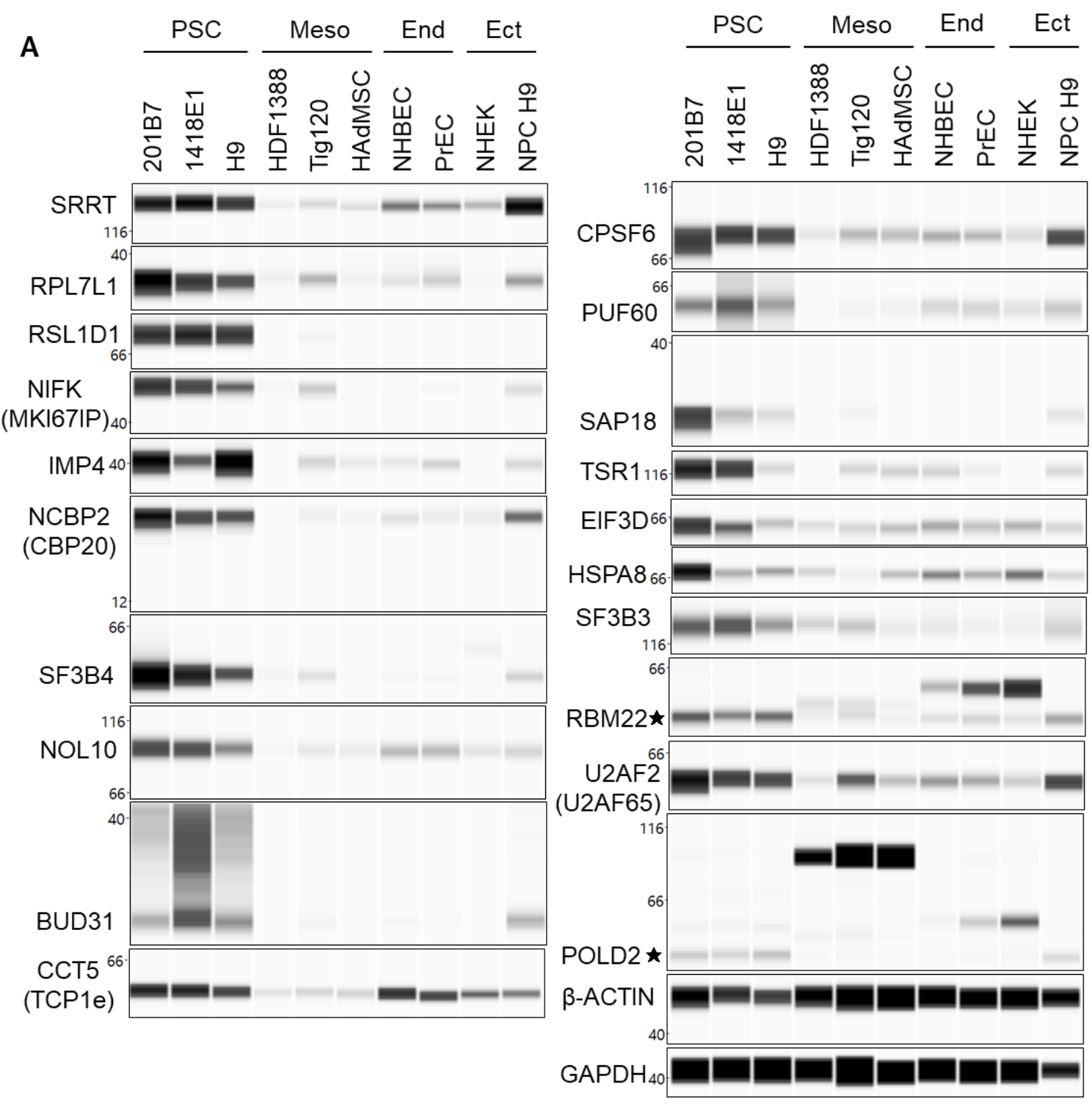

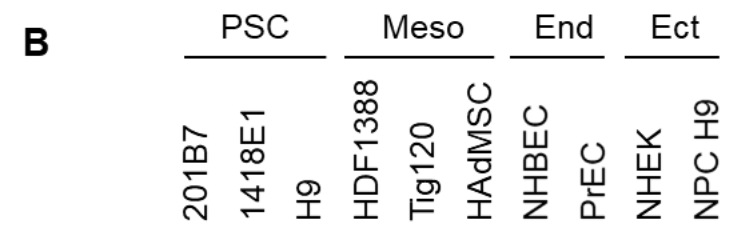

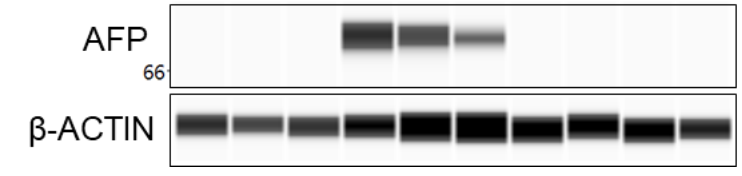


Iwasaki et al., Supplementary Figure 3

C
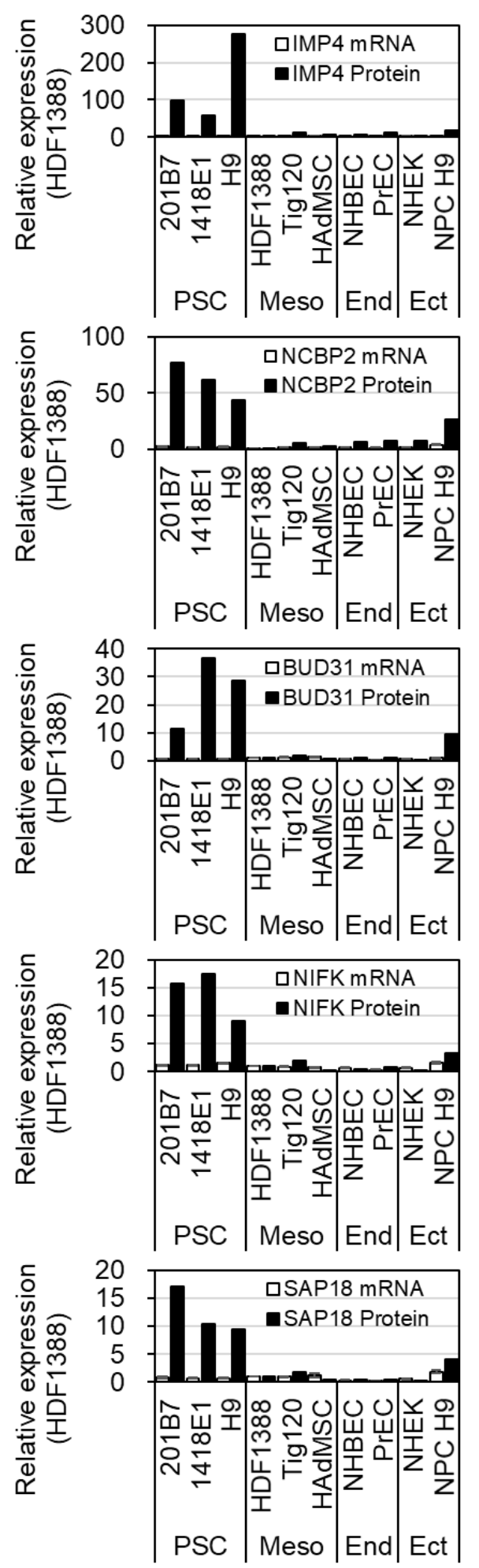
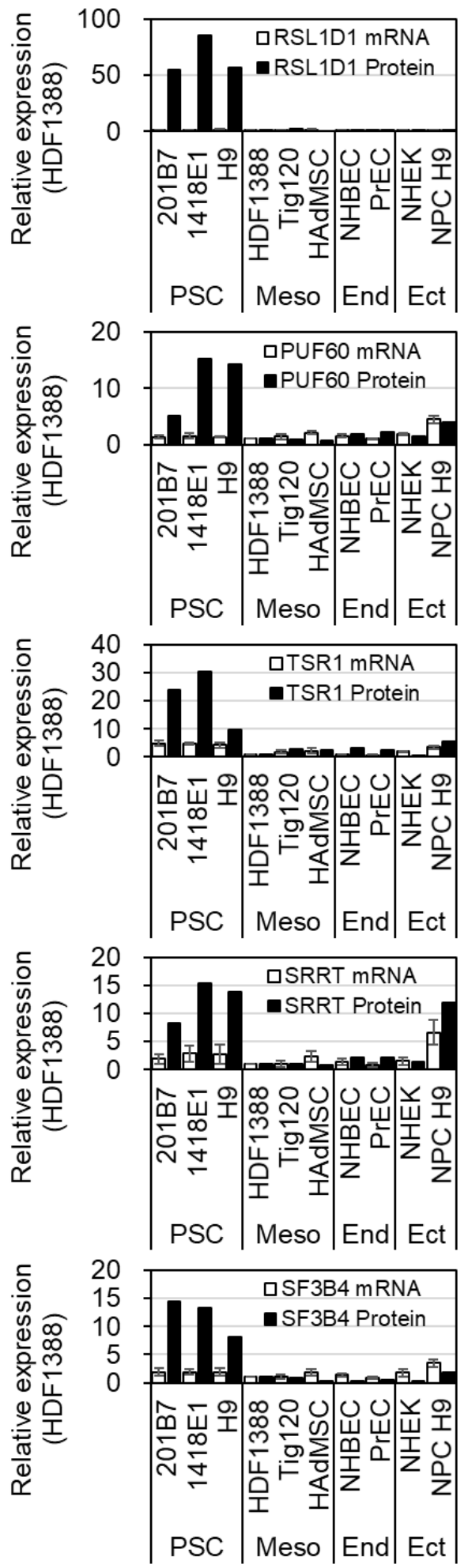
bioRxiv preprint doi: https://doi.org/10.1101/2021.10.18.462967; this version posted October 19,2021. The copyright holder for this preprint (which was not certified by peer review) is the author/funder, who has granted bioRxiv a license to display the preprint in perpetuity. It is made available under aCC-BY-NC-ND 4.0 International license.

Iwasaki et al., Supplementary Figure 3

C

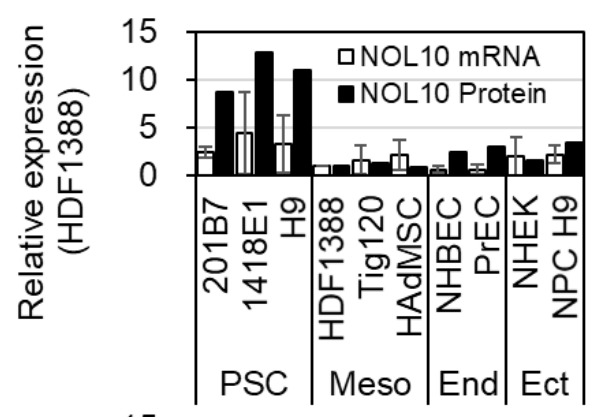

음

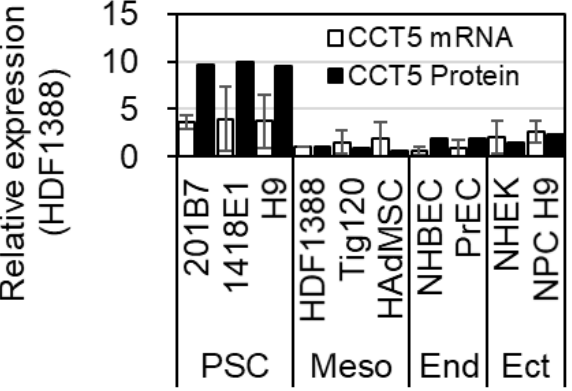

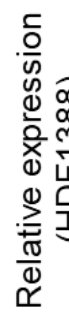

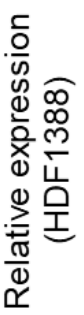

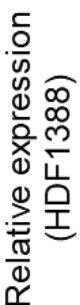

10

PSC $\left|\begin{array}{lr}\text { I } & \text { Meso }\end{array}\right|$ End $\mid$ Ect

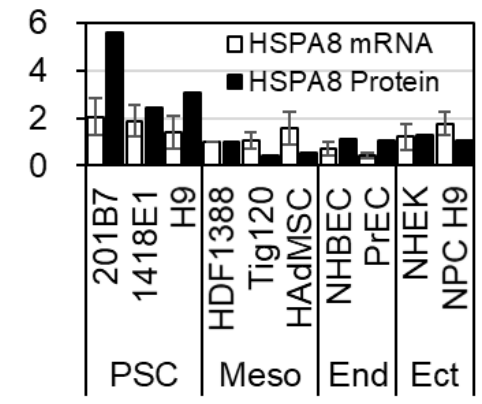

PSC Meso End Ect

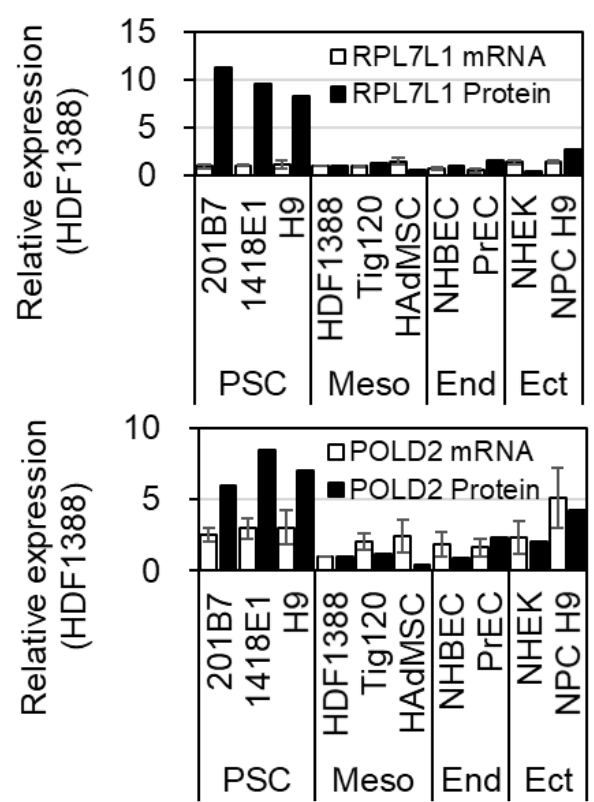

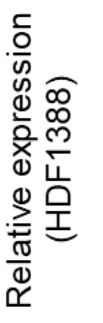

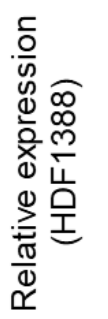

10 口U2AF2 mRNA

5 -

0

뜨 후

$\check{\infty}$

율

PSC Meso |End $\mid$ Ect
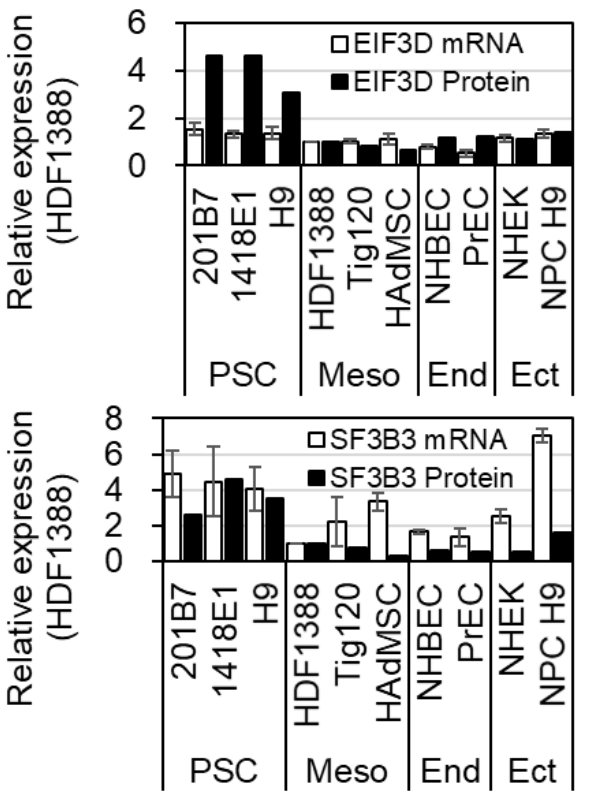
1 Figure S3 | Immunoblotting images and relative expressions of the 20 uPRA genes 2 and one HDF-uPRA gene.

3 A. Immunoblotting images of the 20 essential PSC-uPRA genes. Meso, 4 mesoderm; End, endoderm; Ect, ectoderm. Three cell lines were used for PSCs, 5 three cell lines for mesoderm-derived cells, two cell lines for endoderm-derived cells, 6 and two cell lines for ectoderm-derived cells.

7 B. Immunoblotting image of the 1 essential HDF-uPRA gene (AFP). We could 8 not quantify the mRNA by qRT-PCR because of the low mRNA level.

9 C. Gene expressions measured by qRT-PCR and immunoblotting normalized 10 to HDF-1 (HDF1388). For the mRNA quantification, GAPDH was used for the 11 normalization, and bars represent mean \pm S.D., biological triplicate. For the protein 12 quantification, $\beta$-ACTIN was used for the normalization. 
Iwasaki et al., Supplementary Figure 4

A

$\frac{\text { Proteasome inhibitor }}{\frac{\mathrm{HDF}}{\text { Oh } 2 \mathrm{~h} 6 \mathrm{~h} 8 \mathrm{~h}} \frac{\mathrm{iPSC}}{\text { Oh } 1 \mathrm{~h}}}$

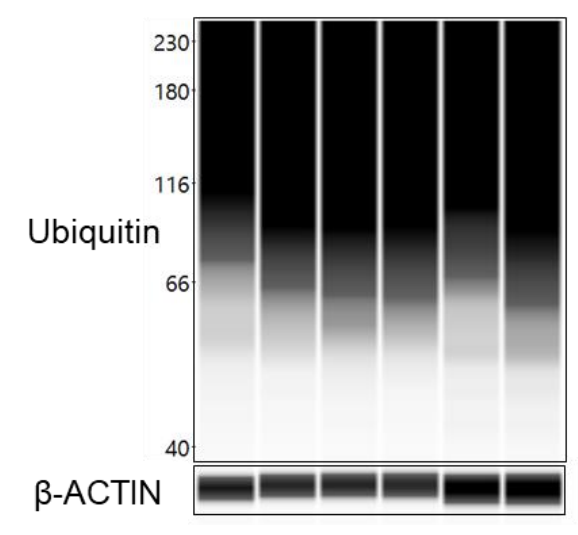

C

$\frac{\text { Proteasome inhibitor }}{\frac{\text { HDF }}{\text { Oh } 2 \mathrm{~h} 24 \mathrm{~h}} \frac{\text { iPSC }}{\text { Oh } 1 \mathrm{~h}}}$

B

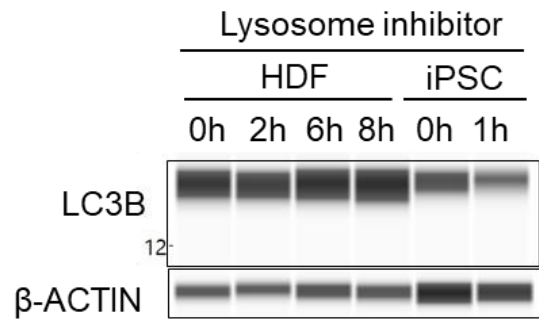

D Lysosome inhibitor

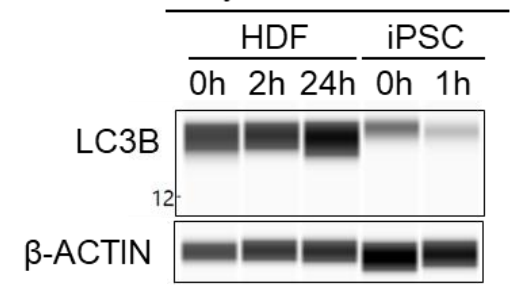


bioRxiv preprint doi: https:/doi.org/10.1101/2021.10.18.462967: this version posted October 192021. The copyright holder for this preprint (which was not certified by peer review) is the author/funder, who has granted bioRxiv a license to display the preprint in perpetuity. It is made available under aCC-BY-NC-ND 4.0 International license.

\section{E}

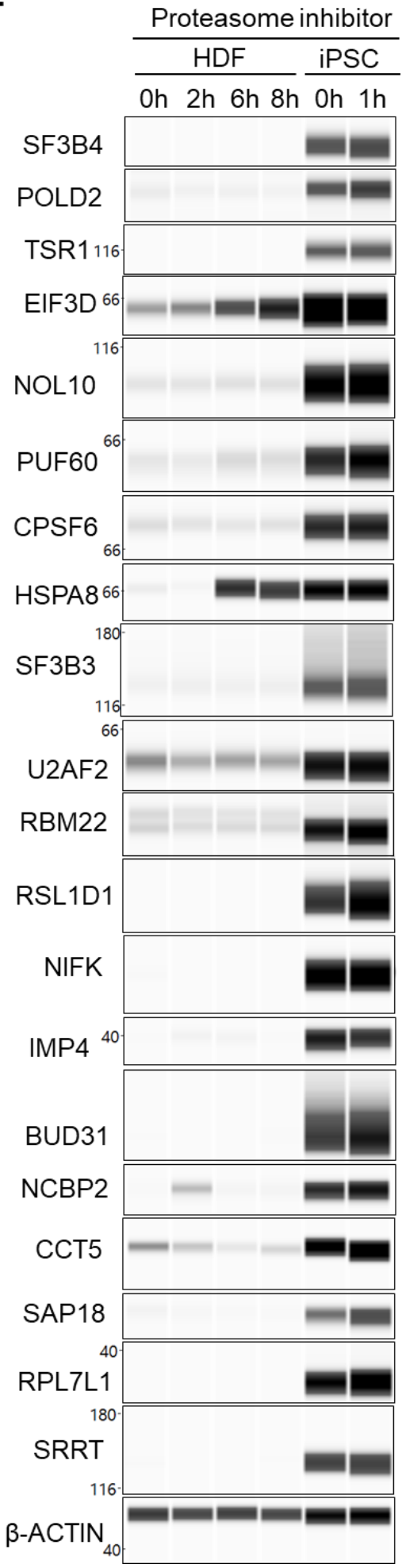

Iwasaki et al., Supplementary Figure 4

$\mathbf{F}$

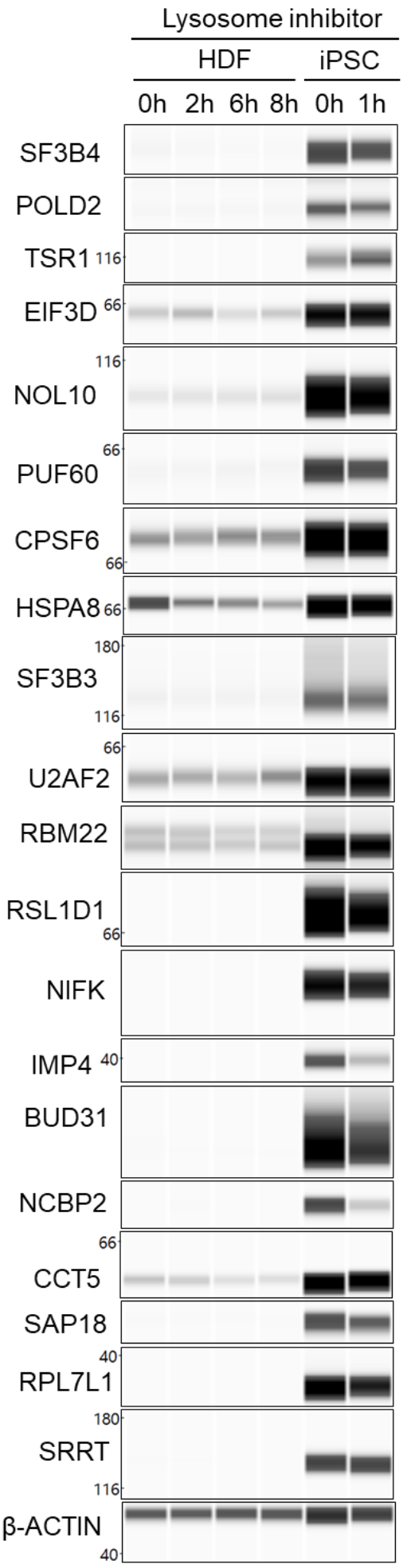


1 Figure S4 | Immunoblotting images of the proteasome inhibitor and lysosomal 2 inhibitor assays.

3 A. Ubiquitin protein levels measured by immunoblotting in iPSC-1 (201B7) and

4 HDF-2 (Tig120) samples treated with a proteasome inhibitor (20 $\square$ M MG-132) for up

5 to 8 hours as a control for the proteasome inhibitor assay.

6 B. LC3B protein levels measured by immunoblotting in iPSC-1 (201B7) and

7 HDF-2 (Tig120) samples treated with lysosomal inhibitors (250 nM Bafilomycin A1

8 and 500 nM Wortmannin) for up to 8 hours as a control for the lysosome inhibitor 9 assay.

10 C. iPSC-1 (201B7) and HDF-2 (Tig120) were treated with $20 \square \mathrm{M} \mathrm{MG-132} \mathrm{for}$ 11 up to 24 hours, and ubiquitin protein levels were measured by immunoblotting.

12 D. iPSC-1 (201B7) and HDF-2 (Tig120) were treated with $250 \mathrm{nM}$ Bafilomycin

13 A1 and $500 \mathrm{nM}$ Wortmannin for up to 24 hours, and LC3B protein levels were 14 measured by immunoblotting.

15 E. The gene expression levels for the 20 essential PSC-uPRA genes were 16 measured by immunoblotting with the same samples used in $A$.

17 F. The gene expression levels for the 20 essential PSC-UPRA genes were 18 measured by immunoblotting with the same samples used in B. 


\section{ACKNOWLEDGMENTS}

15 We would like to thank Y. Fujita and T. Matsushita for the technical assistance, P.

16 Karagiannis for editorial assistance, and S.Takeshima for administrative support.

17 This work was supported by K-CONNEX from the Japan Science and Technology

18 Agency (JST), Core Center for iPS Cell Research from Japan Agency for Medical

19 Research and Development (AMED; JP21bm0104001), AMED-PRIME

20 (21gm6410003h0001 (M.I.)), and the Japanese Society for the Promotion of 
1 Science KAKENHI Grant (JSPS; 19K16104 (M.I.)).

3 AUTHOR CONTRIBUTIONS

4 M.I. and Y.K. performed most of the experiments. T.T generated the program to 5 quantify the MS data. M.Narita performed the microarray. M.Nakagawa., Y.N. and 6 A.O. helped perform the siRNA screening analysis. T.Y. and H.S. performed the 7 mRNA-related experiments. M.I., K.T. and S.Y. designed and directed the research. 8 M.I. analyzed the data and wrote the manuscript with editing by all authors.

\section{DECLARATION OF INTEREST}

11 M.I. is a scientific adviser (without salary) of xFOREST Therapeutics. H.S. is an 12 outside director of aceRNA Technologies, Co., Ltd. K.T. is on the scientific advisory 13 board (without salary) of I Peace, Inc. S.Y. is a scientific advisor (without salary) of 14 iPS Academia Japan. 


\section{REFERENCES}

2 Bentley, D.L. (2014). Coupling mRNA processing with transcription in time and space. Nat Rev Genet

$3 \quad 15,163-175.10 .1038 / \mathrm{nrg} 3662$.

4 Buccitelli, C., and Selbach, M. (2020). mRNAs, proteins and the emerging principles of gene 5 expression control. Nat Rev Genet 21, 630-644. 10.1038/s41576-020-0258-4.

6 Chandrasekaran, V., Juszkiewicz, S., Choi, J., Puglisi, J.D., Brown, A., Shao, S., Ramakrishnan, V., and

7 Hegde, R.S. (2019). Mechanism of ribosome stalling during translation of a poly(A) tail. Nat Struct

8 Mol Biol 26, 1132-1140. 10.1038/s41594-019-0331-x.

9 Chen, Q., and Hu, G. (2017). Post-transcriptional regulation of the pluripotent state. Curr Opin 10 Genet Dev 46, 15-23. 10.1016/j.gde.2017.06.010.

11 D'Orazio, K.N., and Green, R. (2021). Ribosome states signal RNA quality control. Mol Cell 81, 1372-

12 1383. 10.1016/j.molcel.2021.02.022.

13 Darnell, A.M., Subramaniam, A.R., and O'Shea, E.K. (2018). Translational Control through 14 Differential Ribosome Pausing during Amino Acid Limitation in Mammalian Cells. Mol Cell 71, 22915 243.e211. 10.1016/j.molcel.2018.06.041.

16 Dendooven, T., Luisi, B.F., and Bandyra, K.J. (2020). RNA lifetime control, from stereochemistry to 17 gene expression. Curr Opin Struct Biol 61, 59-70. 10.1016/j.sbi.2019.10.002.

18 Gabut, M., Samavarchi-Tehrani, P., Wang, X., Slobodeniuc, V., O'Hanlon, D., Sung, H.K., Alvarez, 19 M., Talukder, S., Pan, Q., Mazzoni, E.O., et al. (2011). An alternative splicing switch regulates 20 embryonic stem cell pluripotency and reprogramming. Cell 147, 132-146. 10.1016/j.cell.2011.08.023.

21 Han, H., Irimia, M., Ross, P.J., Sung, H.K., Alipanahi, B., David, L., Golipour, A., Gabut, M., Michael, 22 I.P., Nachman, E.N., et al. (2013). MBNL proteins repress ES-cell-specific alternative splicing and 23 reprogramming. Nature 498, 241-245. 10.1038/nature12270.

24 Hentze, M.W., Castello, A., Schwarzl, T., and Preiss, T. (2018). A brave new world of RNA-binding 25 proteins. Nat Rev Mol Cell Biol 19, 327-341. 10.1038/nrm.2017.130.

26 Kainov, Y.A., and Makeyev, E.V. (2020). A transcriptome-wide antitermination mechanism sustaining 27 identity of embryonic stem cells. Nat Commun 11, 361. 10.1038/s41467-019-14204-z.

28 Khan, Z., Ford, M.J., Cusanovich, D.A., Mitrano, A., Pritchard, J.K., and Gilad, Y. (2013). Primate 29 transcript and protein expression levels evolve under compensatory selection pressures. Science 342, $30 \quad 1100-1104.10 .1126 /$ science.1242379.

31 Lee, A.S., Kranzusch, P.J., Doudna, J.A., and Cate, J.H. (2016). eIF3d is an mRNA cap-binding protein 32 that is required for specialized translation initiation. Nature 536, 96-99. 10.1038/nature18954.

33 Leppek, K., Fujii, K., Quade, N., Susanto, T.T., Boehringer, D., Lenarčič, T., Xue, S., Genuth, N.R., 
1 Ban, N., and Barna, M. (2020). Gene- and Species-Specific Hox mRNA Translation by Ribosome 2 Expansion Segments. Mol Cell 80, 980-995.e913. 10.1016/j.molcel.2020.10.023.

3 Lu, R., Markowetz, F., Unwin, R.D., Leek, J.T., Airoldi, E.M., MacArthur, B.D., Lachmann, A., Rozov,

4 R., Ma'ayan, A., Boyer, L.A., et al. (2009). Systems-level dynamic analyses of fate change in murine

5 embryonic stem cells. Nature 462, 358-362. 10.1038/nature08575.

6 Matsumoto, M., Matsuzaki, F., Oshikawa, K., Goshima, N., Mori, M., Kawamura, Y., Ogawa, K.,

7 Fukuda, E., Nakatsumi, H., Natsume, T., et al. (2017). A large-scale targeted proteomics assay

8 resource based on an in vitro human proteome. Nat Methods 14, 251-258. 10.1038/nmeth.4116.

9 Mauger, D.M., Cabral, B.J., Presnyak, V., Su, S.V., Reid, D.W., Goodman, B., Link, K., Khatwani, N.,

10 Reynders, J., Moore, M.J., and McFadyen, I.J. (2019). mRNA structure regulates protein expression

11 through changes in functional half-life. Proc Natl Acad Sci U S A 116, 24075-24083.

$12 \quad 10.1073 /$ pnas.1908052116.

13 Ohta, S., Nishida, E., Yamanaka, S., and Yamamoto, T. (2013). Global splicing pattern reversion 14 during somatic cell reprogramming. Cell Rep 5, 357-366. 10.1016/j.celrep.2013.09.016.

15 Palazzo, A.F., and Lee, E.S. (2018). Sequence Determinants for Nuclear Retention and Cytoplasmic 16 Export of mRNAs and lncRNAs. Front Genet 9, 440. 10.3389/fgene.2018.00440.

17 Pelletier, J., Thomas, G., and Volarević, S. (2018). Ribosome biogenesis in cancer: new players and 18 therapeutic avenues. Nat Rev Cancer 18, 51-63. 10.1038/nrc.2017.104.

19 Pohl, C., and Dikic, I. (2019). Cellular quality control by the ubiquitin-proteasome system and 20 autophagy. Science 366, 818-822. 10.1126/science.aax3769.

21 Roumeliotis, T.I., Williams, S.P., Gonçalves, E., Alsinet, C., Del Castillo Velasco-Herrera, M., Aben, 22 N., Ghavidel, F.Z., Michaut, M., Schubert, M., Price, S., et al. (2017). Genomic Determinants of 23 Protein Abundance Variation in Colorectal Cancer Cells. Cell Rep 20, 2201-2214. 24 10.1016/j.celrep.2017.08.010.

25 Salomonis, N., Schlieve, C.R., Pereira, L., Wahlquist, C., Colas, A., Zambon, A.C., Vranizan, K., 26 Spindler, M.J., Pico, A.R., Cline, M.S., et al. (2010). Alternative splicing regulates mouse embryonic 27 stem cell pluripotency and differentiation. Proc Natl Acad Sci U S A 107, 10514-10519. 28 10.1073/pnas.0912260107.

29 Teixeira, F.K., and Lehmann, R. (2019). Translational Control during Developmental Transitions. 30 Cold Spring Harb Perspect Biol 11. 10.1101/cshperspect.a032987.

31 Tresenrider, A., Morse, K., Jorgensen, V., Chia, M., Liao, H., van Werven, F.J., and Ünal, E. (2021). 32 Integrated genomic analysis reveals key features of long undecoded transcript isoform-based gene 33 repression. Mol Cell 81, 2231-2245.e2211. 10.1016/j.molcel.2021.03.013. 
1 Umemura, Y., Koike, N., Ohashi, M., Tsuchiya, Y., Meng, Q.J., Minami, Y., Hara, M., Hisatomi, M.,

2 and Yagita, K. (2017). Involvement of posttranscriptional regulation of Clock in the emergence of

3 circadian clock oscillation during mouse development. Proc Natl Acad Sci U S A 114, E7479-E7488.

$4 \quad 10.1073 /$ pnas. 1703170114.

5 Wang, D., Eraslan, B., Wieland, T., Hallström, B., Hopf, T., Zolg, D.P., Zecha, J., Asplund, A., Li,

6 L.H., Meng, C., et al. (2019). A deep proteome and transcriptome abundance atlas of 29 healthy

7 human tissues. Mol Syst Biol 15, e8503. 10.15252/msb.20188503. 